TRANSACTIONS OF THE

AMERICAN MATHEMATICAL SOCIETY

Volume 322, Number 1, November 1990

\title{
MEAN-SQUARE APPROXIMATION BY POLYNOMIALS ON THE UNIT DISK
}

\author{
THOMAS L. KRIETE III AND BARBARA D. MACCLUER
}

\begin{abstract}
We investigate $P^{2}(\mu)$, the closure in $L^{2}(\mu)$ of the complex polynomials, for certain measures $\mu$ on the closed unit disk in the complex plane. Specifically, we present a condition on $\mu$ which guarantees that $P^{2}(\mu)$ decomposes into a natural direct sum.
\end{abstract}

\section{INTRODUCTION}

Let $\mu$ be a positive finite Borel measure with compact support in the complex plane. Much interesting analysis has resulted from attempts to understand the structure of $P^{2}(\mu)$, the closure in $L^{2}(\mu)$ of the polynomials. Work of Carleman [Ca], Keldyš [Kd] and Brennan [B1, B2], among others, has dealt with the case $\mu=W \chi_{U} d x d y$, where $U$ is a simply connected domain and $W>0$ a measurable function on $U$. (Here, and throughout, $\chi_{E}$ denotes the characteristic function of the set $E$.) In this setting the point of view has been to obtain conditions on $U$ and $W$ which guarantee that $P^{2}(\mu)$ be exactly the set of analytic functions in $L^{2}(U, W d x d y)$. A related question for general $\mu$ is whether $P^{2}(\mu)$ will always possess a bounded point evaluation if $P^{2}(\mu) \neq L^{2}(\mu)$ [B1, Hr3, Tr2].

In this paper we will be concerned with measures of the form $\mu=\nu+w d \theta$ where $\nu$ is carried by the open unit disc $D, d \theta$ is Lebesgue measure on the unit circle $\partial D$, and $w \geq 0$ is in $L^{1}=L^{1}(d \theta)$. In the more general situation in which the part of $\mu$ carried by $\partial D$ has a Lebesgue decomposition $w d \theta+\mu_{s}$ with $\mu_{s} \perp d \theta, P^{2}(\mu)$ will contain $L^{2}\left(\mu_{s}\right)$ as a direct summand [Cl]. Hence, there is no loss of generality in assuming at the outset that $\mu=\nu+w d \theta$. If $\log w \in L^{1}$, then $P^{2}(\mu)$ is a nice space of analytic functions whose structure is like that of the Hardy space $H^{2}[\mathrm{Cl}$. Thus we consider only the case where

$$
\int_{\partial D} \log w d \theta=-\infty
$$

Received by the editors July 8, 1987 and, in revised form, January 27, 1989.

1980 Mathematics Subject Classification (1985 Revision). Primary 30E10; Secondary 46E20, 30B60.

Both authors were supported in part by the National Science Foundation.

Part of this work was done while the first author was visiting the University of South Carolina, and he thanks the U.S.C. Faculty and Staff for their hospitality. He also thanks the University of Virginia for supporting him during this period as a Sesquicentennial Associate. 
Under this hypothesis, $P^{2}(w d \theta)=L^{2}(w d \theta)$ by Szegö's Theorem [H, p. 49]. Our results give conditions under which $P^{2}(\mu)$ splits, that is, decomposes as a direct sum $P^{2}(\nu) \oplus L^{2}(w d \theta)$. This means, of course, that the natural isometry $f \rightarrow \chi_{D} f+\chi_{\partial D} f$ maps $P^{2}(\mu)$ onto $P^{2}(\nu) \oplus L^{2}(w d \theta)$. We will suppose that the part of $\mu$ carried by $D$ is rotation invariant and absolutely continuous with respect to Lebesgue area measure $d A$ on $D$. In particular, we assume $\nu=G(r) r d r d \theta \equiv G d A$, where $G$ is continuous and positive on [0,1). Then $P^{2}(\nu)$ is the space of analytic functions $f=\sum_{0}^{\infty} a_{n} z^{n}$ on $D$ with

$$
\int_{D}|f|^{2} G d A=2 \pi \sum_{0}^{\infty}\left|a_{n}\right|^{2} p_{n}<\infty
$$

where $p_{n}=\int_{0}^{1} r^{2 n+1} G(r) d r$. Hence if splitting occurs in this case, $P^{2}(\mu)$ will be completely understood.

The general question of splitting, under a variety of conditions on $\nu$ and $w$, has been studied by various authors [Ke, $\mathrm{N}, \mathrm{Ha}, \mathrm{Hr} 1, \mathrm{Hr} 2, \mathrm{Kr} 1, \mathrm{Kr} 2, \mathrm{~V} 1$, $\mathrm{V} 2, \mathrm{~B} 2]$. If $\nu$ has compact support in $D$ (and (1.1) holds), then $P^{2}(\mu)$ splits [Krl]. Based on this, one might expect splitting to occur if $\nu=G d A$ as above and $G(r)$ decreases to zero sufficiently rapidly as $r \uparrow 1$. Indeed, it was shown in $[\mathrm{Krl}]$ that the relevant measure of how fast $G$ must decrease is

$$
\left.\int_{1-\delta}^{1} \log \log \frac{1}{G(r)} d r \quad \text { (some small } \delta\right) .
$$

Let us always use the term $\operatorname{arc}$ to mean a nonempty open arc in $\partial D$.

Theorem D. Suppose the integral (1.2) converges (and $G$ satisfies a mild regularity condition). If $\log w \in L^{1}(I)$ for some arc $I \subset \partial D$, then $P^{2}(\mu)$ does not split.

This theorem, which is proved in $\S 7$ below, improves results from [ $\mathrm{Kr} 1]$ and [Tr1]. The best result in the converse direction (indeed, it is best possible) is due to A. L. Vol' berg and is based on his work on quasianalyticity [V3, VE].

Theorem 1.1 (Vol' berg). Suppose that the integral (1.2) diverges (and $G$ satisfies mild regularity conditions). If $\log w \notin L^{1}$, then $P^{2}(\mu)$ splits.

This result can be derived from Theorem 1 in [VE] in the same way that Theorem 5 and Corollary 2 in [Krl] are deduced from the Levinson-Beurling Theorem. In this paper, then, we will be concerned only with the situation in which $G$ decreases to zero slowly enough that the integral (1.2) is finite, or even with the case where $G$ does not decrease at all, e.g., $G(r) \equiv 1$. Note that this includes the standard Bergman space weights $G(r)=(1-r)^{\alpha}, \alpha>-1$, weights which decay exponentially like

$$
G(r)=\exp \left(-\left(\frac{1}{1-r}\right)^{\alpha}\right), \quad \alpha>0,
$$


and certain "double exponential" weights like

$$
G(r)=\exp \left(-\exp \left(\frac{1}{1-r}\right)^{\alpha}\right), \quad 0<\alpha<1 .
$$

The demarcation indicated by the convergence or divergence of (1.2) (which we might call the "log log cutoff") clearly separates very different types of behavior. There is also a "log cutoff" signaled approximately by whether or not

$$
\int_{1-\delta}^{1} \log \frac{1}{G(r)} d r \quad(\delta \text { small })
$$

converges. For example, in the exponential weights (1.3) this cutoff occurs at $\alpha=1$. That convergence or divergence in (1.4) marks a change in behavior is suggested by the work of Trent [Tr1], and by results of Hruščëv [Hr1, Hr2] and Kegegan [Ke] about measures $\mu$ with $w=\chi_{E}$, where $E$ is a measurable subset of $\partial D$. Hruščèv and Kegegan develop their approximation results in terms of certain Banach space norms, but it is a simple matter to recast them in terms of $L^{2}(\mu)$-convergence provided $\mu$ is of the special type considered here (see Theorems 3.1, 4.1 and 10.1 in [Hr2]). Their criterion for splitting involves a generalization of the well-known idea of a Carleson set. Suppose $F$ is a proper closed subset of $\partial D$, so that the complement $\partial D \backslash F$ is the union of a (possibly finite) sequence $\left\{I_{k}\right\}$ of disjoint open arcs. Let us assume that $G(r)<1$ provided $r$ is near 1 . Then $F$ is a $G$-Carleson set provided

$$
\sum\left|I_{k}\right| \log \frac{1}{G\left(1-\left|I_{k}\right|\right)}<\infty
$$

(Here and throughout $|E|$ denotes the Lebesgue measure of the set $E \subset \partial D$.)

Theorem 1.2 [Hr2]. Suppose that $(1-r) \log \left(G(r)^{-1}\right)$ is nonincreasing for $r$ near 1 and that $G$ satisfies a mild regularity hypothesis. Let $\mu=G d A+\chi_{E} d \theta$ where $E$ is a measurable subset of $\partial D$. In order for $P^{2}(\mu)$ to split it is sufficient that there exist no closed G-Carleson set $F$ of positive measure with $|F \backslash E|=0$. Conversely, if $G$ satisfies an additional technical hypothesis (which in particular requires that

$$
\int_{1-\delta}^{1} \log \frac{1}{G(r)} d r<\infty
$$

for small $\delta$ ), then the above sufficient condition for splitting is also necessary.

This theorem applies to the standard Bergman space weights $(1-r)^{\alpha}$, with $\alpha \geq 0$, as well as the weights (1.3) with $0<\alpha<1$. The "cutoff" case $\alpha=1$ of these latter weights is handled by the sufficient condition from Theorem 1.2 together with Theorem $\mathrm{D}$; for this case the $G$-Carleson criterion collapses dramatically: If

$$
G(r)=\exp \left(-\frac{1}{1-r}\right)
$$

and $\mu=G d A+\chi_{E} d \theta$, then $P^{2}(\mu)$ splits if and only if $|I \backslash E|>0$ for every arc $I \subset \partial D$. Moreover, Theorem D implies that no further change occurs (at 
least when $w=\chi_{E}$ ) all the way down to the $\log \log$ cutoff (see the proof of Corollary 7.1 below).

Our purpose in this paper is to pass from characteristic functions $\chi_{E}$ to arbitrary nonnegative weights $w \in L^{1}$ in the setting where the integral (1.2) converges. That an interesting theory resides in this context was foreshadowed by an example of $\mathrm{Vol}^{\prime}$ berg of a weight $w$ with $w>0$ a.e. such that $P^{2}(d A+w d \theta)$ splits (see the editors' commentary in [ $\left.\mathrm{Kr} 2\right]$ which also includes a more refined example of Hruščèv). Theorem D above implies that for splitting to occur here (unlike the case where (1.2) diverges), $w$ must be small on sets which are generously spread throughout $\partial D$. Indeed, Trent's early version of Theorem D [ $\operatorname{Tr} 1$ ], in which convergence of the integral (1.4) is required, convinced us that the mechanism of splitting hinges on a pervasive failure (in some sense) of local integrability for $\log w$. We will here confirm this intuition by presenting conditions sufficient for splitting which are strengthenings of the requirement that for every arc $I \subset \partial D, \log w$ is not in weak $L^{1}(I)$. To say that $\log w \notin$ weak $L^{1}(I)$ is equivalent to saying that

$$
\sup _{0<\varepsilon<t_{0}} \Omega_{I}(\varepsilon) \log \frac{1}{\varepsilon}=+\infty \quad\left(t_{0} \text { a fixed positive number }\right),
$$

where $\Omega_{I}(\varepsilon)$ is the proportion of $I$ on which $w \leq \varepsilon$, that is

$$
\Omega_{I}(\varepsilon)=\frac{1}{|I|}\left|\left\{e^{i x} \in I: w\left(e^{i x}\right) \leq \varepsilon\right\}\right| .
$$

We will often work with a uniformized version of $\Omega_{I}(\varepsilon)$ defined by

$$
\Omega(\delta, \varepsilon) \equiv \inf _{|I|=\delta} \Omega_{I}(\varepsilon),
$$

where, as indicated, the infimum is over all arcs $I \subset \partial D$ with $|I|=\delta$. Note that $0 \leq \Omega(\delta, \varepsilon) \leq 1$. If $w>0$ a.e. and $\delta$ is fixed, then $\Omega(\delta, \varepsilon) \rightarrow 0$ as $\varepsilon \rightarrow 0$. Also, if $\varepsilon$ is fixed and $w$ is greater than $\varepsilon$ on a set of positive measure, then $\Omega(\delta, \varepsilon) \rightarrow 0$ as $\delta \rightarrow 0$. (To see this consider arcs centered at a point $e^{i \theta}$ which is a Lebesgue point for $\chi_{\{w \leq \varepsilon\}}$ but for which $\chi_{\{w \leq \varepsilon\}}\left(e^{i \theta}\right)=0$.) We now state our theorem which is applicable if $G(r)=(1-r)^{\alpha}$.

Theorem A. Let $\mu=(1-|z|)^{\alpha} d A+w d \theta$ where $\alpha>-1$. Suppose $\gamma>0$ and let $f(t)=e^{-1 / t^{\prime}}$. If

$$
\sup _{0<\varepsilon<1} \Omega(f(\varepsilon), \varepsilon) \log \frac{1}{\varepsilon}=+\infty,
$$

then $P^{2}(\mu)=P^{2}\left((1-|z|)^{\alpha} d A\right) \oplus L^{2}(w d \theta)$.

For the weights $G(r)$ given by (1.3), $0<\alpha \leq 1$, we have Theorem B, in which one simply replaces the function $f(t)$ from Theorem A by

$$
f(t)=\exp \left(-\left(\log \frac{1}{t}\right)^{(\alpha+1) / \alpha}\right) \text {. }
$$


In general the hypothesis

$$
\sup _{0<\varepsilon<t_{0}} \Omega(f(\varepsilon), \varepsilon) \log \frac{1}{\varepsilon}=+\infty
$$

becomes easier to satisfy the more slowly $f(t)$ tends to zero with $t$. Theorems $\mathrm{A}$ and $\mathrm{B}$ are reasonably sharp in that one cannot replace the given function $f(t)$ with anything decreasing very much more slowly without having the theorems become false. However, just beyond the log cutoff one can use any function $f(t)$ and still achieve splitting.

Theorem $\mathbf{C}^{\prime}$. Let $\mu=G d A+w d \theta$ where $G(r)$ is decreasing for $r$ near 1 and satisfies

$$
\lim _{r \uparrow 1}(1-r) \log \frac{1}{G(r)}=+\infty .
$$

If there exist sequences $\left\{\delta_{n}\right\}$ and $\left\{\varepsilon_{n}\right\}$ of positive numbers, both tending to zero, so that

$$
\lim _{n \rightarrow \infty} \Omega\left(\delta_{n}, \varepsilon_{n}\right) \log \frac{1}{\varepsilon_{n}}=+\infty,
$$

then $P^{2}(\mu)=P^{2}(G d A) \oplus L^{2}(w d \theta)$.

In this result and a more general version (Theorem $\mathrm{C}$ ), the hypothesis on $w$ may be thought of as saying that $\log w$ uniformly fails to be locally in weak $L^{1}$.

It is clear that we are on target in looking at the local behavior of $\log w$. Our notion of "local", however, is tied to arcs, and although we establish a loose link with $G$-Carleson sets in $\S 8$, a complete solution to our problem (when the integral (1.4) converges) awaits a more definitive connection. On the other hand, when $G$ lies between the $\log$ and $\log \log$ cutoffs, $G$-Carleson sets disappear from the picture and arcs surely provide the right notion of "local". In this case our results are close to definitive, though the problem of $L^{1}$ vs. weak $L^{1}$ is unresolved. Thus, interesting questions remain to be answered. See $\S 9$ for further comments.

The proofs of our theorems involve several different steps which we outline here. It is well known that $P^{2}(\mu)$. splits if and only if $\chi_{D}$ lies in $P^{2}(\mu)$. Thus we will be concerned with the quantity

$$
\inf \left\{\int\left|\chi_{D}-p\right|^{2} d \mu: p \in P^{2}(\mu)\right\} \text {. }
$$

For our purposes it will turn out to be sufficient to consider only those functions $p$ in $P^{2}(\mu)$ of a very special type, specifically $p=h \circ B$, where $B$ is an inner function arising from a Cayley inner function associated to the weight function $w$, and $h$ is an outer function in $H^{\infty}$ of a particularly simple form. In $\S 2$ we discuss the construction of those Cayley inner functions which are needed for our argument. In $\S 3$ we present the strategy for estimating the distance from $\chi_{D}$ to $P^{2}(\mu)$. It will become clear that good estimates for this 
distance (which we ultimately show is zero) require information about the norm of a certain composition operator $T$ induced by our inner function $B$ and acting on a certain weighted Dirichlet space $\mathscr{D}$. Similar weighted Dirichlet spaces have proved to be appropriate settings for recent work on compactness of composition operators [MS, Sh]. Our estimate of the norm of $T$ requires knowledge of the size of $B$ near $\partial D$ (the subject of $\S 4$ ) and is then deduced as a corollary of the main result of $\S 5$, a norm estimate for arbitrary composition operators on $\mathscr{D}$. We believe the results of $\S 5$ are of independent interest. The norm estimate itself depends on a change-of-variable formula and an associated "counting function," a strategy introduced by Shapiro in the pioneering paper $[\mathrm{Sh}]$.

In $\S 6$ we present Theorems $\mathrm{A}$ and $\mathrm{B}$ (deducing both from the more general Theorem 6.2) and show that there is an abundant supply of weight functions $w$ satisfying our hypotheses; $\S 7$ is devoted to Theorems $C, C^{\prime}$ and D. The sharpness of our results is considered in $\S 8$ and we conclude in $\S 9$ with some directions for further research.

We close these introductory remarks with a fact that will be used several times in the sequel. Let $G(r)$ be any weight of the type we have been considering (it is enough to assume $G$ is positive and integrable on $[0,1)$ ). Let $R$ denote the annulus $\{z: \rho<|z|<1\}$ where $0<\rho<1$. It is then easy to see that there exists $c>0$ such that

$$
\int_{D}|u|^{2} G d A \leq c \int_{R}|u|^{2} G d A
$$

for all functions $u$ analytic on $D$. Moreover, let $\mu_{1}=G_{1} d A+w d \theta$ and $\mu_{2}=G_{2} d A+w d \theta$ be two measures with the same boundary weight $w$ and suppose that $G_{1}(r) \leq a G_{2}(r)$ for some $a>0$ and all $r$ in $(\rho, 1)$. It follows from (1.6) that $P^{2}\left(\mu_{1}\right)$ splits if $P^{2}\left(\mu_{2}\right)$ splits. Thus $G(r)$ influences splitting in $P^{2}(G d A+w d \theta)$ only through its behavior for $r$ near 1 .

\section{Cayley inner functions and the definition of $B$}

In this section we recall how the theory of Cayley inner functions of Rosenblum and Rovnyak [RR] associates to a particular measurable set $\Delta \subset \partial D$ an inner function $B$ with certain mapping properties. The set $\Delta$ will be associated with a positive number $\varepsilon$, a positive integer $n$ and the function $w$; we will describe it shortly. For the moment we merely suppose that $0<|\Delta|<2 \pi$.

The corresponding Cayley inner function $\Psi$ is defined by

$$
\Psi(z)=\exp \left\{\frac{i}{2} \int_{\Delta} \frac{e^{i x}+z}{e^{i x}-z} d x\right\} .
$$

The properties of $\Psi$ listed below can be found in [RR]. The function $\Psi$ maps $D$ into the upper half-plane $\{z: \operatorname{Im} z>0\}$; the nontangential boundary-value function $\Psi\left(e^{i \theta}\right)$ exists a.e. on $\partial D$, and satisfies $\Psi\left(e^{i \theta}\right)<0$ a.e. on $\Delta$ and $\Psi\left(e^{i \theta}\right) \geq 0$ a.e. on $\partial D \backslash \Delta$. Here and throughout we write $s=|\Delta|$ and 
$\Gamma=\left\{e^{i x}: 0<x<s\right\}$. We put

$$
\lambda(\zeta)=\frac{\zeta-e^{i s / 2}}{\zeta-e^{-i s / 2}}
$$

a conformal map of the upper half-plane onto $D$. One checks that $\lambda$ maps $(-\infty, 0)$ onto $\Gamma$ and $[0, \infty)$ into $\partial D \backslash \Gamma$. We define $B=\lambda \circ \Psi$. Note that $B$ is an inner function with $B(0)=0$ such that $B\left(e^{i \theta}\right) \in \Gamma$ for almost all $e^{i \theta} \in \Delta$, and $B\left(e^{i \theta}\right) \in \partial D \backslash \Gamma$ for almost all $e^{i \theta} \in \partial D \backslash \Delta$.

Now we are ready to describe $\Delta$. Suppose that $n$ is a positive integer, $n \geq 2$, and let $\varepsilon>0$. Select arcs $I_{1}, \ldots, I_{n}$ equally spaced around $\partial D$ and each of length $\frac{1}{n}$. Suppose that each $I_{k}$ contains a measurable subset $\Delta_{k}$ of positive measure with $w \leq \varepsilon$ on $\Delta_{k}$. Suppose further that the sets $\Delta_{1}, \ldots, \Delta_{n}$ all have the same measure. For the remainder of the paper we will take $\Delta=\bigcup_{k=1}^{n} \Delta_{k}$ and associate $B$ to $\Delta$ as above. Note that $0<|\Delta| \leq 1$. In the course of proving Theorems $\mathrm{A}, \mathrm{B}$, and $\mathrm{C}$, we will use our hypotheses on $w$ to show that such a set $\Delta$ indeed exists and that $|\Delta|$ is not too small.

\section{THE DISTANCE FROM $\chi_{D}$ TO $P^{2}(\mu)$}

Here we estimate the distance from $\chi_{D}$ to $P^{2}(\mu)$; eventually we will show that this distance is zero when our hypotheses hold. Throughout this section we will assume that $\varepsilon, \Delta, \Gamma$ and $B$ are related as in $\S 2$. We begin with an idea of Nikol' skii and Vol 'berg (see editors' comments in [Kr2]) which they used with $z^{n}$ replacing $B$. Let $h$ be an outer function on $D$ such that $h(0)=1$ and $|h|$ is a.e. constant on each of the arcs $\Gamma$ and $\partial D \backslash \Gamma$. We will approximate $\chi_{D}$ by $g(z)=h(B(z))$. Since $g \in H^{\infty}$, the space of bounded analytic functions on $D, g$ is easily seen to lie in $P^{2}(\mu)$. We also have $g\left(e^{i \theta}\right)=h\left(B\left(e^{i \theta}\right)\right)$ a.e., (see [Ry]). Now if $|h|^{2}=M$ a.e. on $\Gamma$ and $|h|^{2}=t$ a.e. on $\partial D \backslash \Gamma$, the mapping properties of $B$ guarantee that $|g|^{2}=M$ a.e. on $\Delta$ and $|g|^{2}=t$ a.e. on $\partial D \backslash \Delta$. Clearly we have

$$
\int\left|\chi_{D}-g\right|^{2} d \mu=\int_{\Delta}|g|^{2} w d \theta+\int_{\partial D \backslash \Delta}|g|^{2} w d \theta+\int_{D}|1-g|^{2} G d A .
$$

Vol' berg used this decomposition with $g(z)=h\left(z^{n}\right)$ to construct a positive $w$ for which $P^{2}(d A+w d \theta)$ splits.

Now $w \leq \varepsilon$ on $\Delta$ and $|\Delta| \leq 1$, hence the sum of the first two terms on the right side of (3.1) cannot exceed

$$
M \varepsilon+t \int_{\partial D} w d \theta
$$

Let us investigate the third term. The first step is to replace the norm in $P^{2}(G d A)$ by a "weighted Dirichlet norm". We put

$$
H(r)=\int_{r}^{1}\left[\int_{x}^{1} G(s) d s\right] d x, \quad 0 \leq r<1
$$


The next lemma is a generalization of the well-known result [MS] that if $u$ is analytic in $D$ with $u(0)=0$, then $\int_{D}|u|^{2}(1-|z|)^{\alpha} d A$ and $\int_{D}\left|u^{\prime}\right|^{2}(1-|z|)^{\alpha+2} d A$ are comparable.

Lemma 3.1. Let $u$ be analytic in $D$ with $u(0)=0$. Then

$$
\int\left|u^{\prime}\right|^{2} H d A \leq \int|u|^{2} G d A \leq 6 \int\left|u^{\prime}\right|^{2} H d A,
$$

where it is understood that the integrals can be infinite.

Proof. Let us put

$$
p_{n}=\int_{0}^{1} r^{2 n+1} G(r) d r, \quad q_{n}=\int_{0}^{1} r^{2 n+1} H(r) d r .
$$

Integrating by parts twice gives $p_{n}=2 n(2 n+1) q_{n-1} \leq 6 n^{2} q_{n-1}$. Now if $u=\sum_{1}^{\infty} a_{n} z^{n}$,

$$
\int|u|^{2} G d A=2 \pi \sum_{1}^{\infty}\left|a_{n}\right|^{2} p_{n} \leq 2 \pi \sum_{1}^{\infty} 6 n^{2}\left|a_{n}\right|^{2} q_{n-1}=6 \int\left|u^{\prime}\right|^{2} H d A .
$$

The other inequality follows similarly.

The weighted Dirichlet norm $\left(\int\left|u^{\prime}\right|^{2} H d A\right)^{1 / 2}$ is not quite what we want, and we proceed one step further. Let us suppose that $F$ is a positive Lebesgue measurable function on $(0, \infty)$ satisfying the following:

$$
\begin{gathered}
H(r) \leq F\left(\log \frac{1}{r}\right), \quad 0<r<1, \\
\quad \int_{0}^{\infty} F(t) e^{-2 t} d t<\infty, \\
F(t) / t \text { is nondecreasing on }(0, \infty) .
\end{gathered}
$$

We note that $F(\log (1 /|z|)) d A$ is a finite measure on $D$, because $(3.2 \mathrm{~b})$ merely says $\int_{0}^{1} F\left(\log \frac{1}{r}\right) r d r<\infty$.

Let us denote by $\mathscr{D}$ the collection of all functions $u$ analytic on $D$ with $u(0)=0$ and satisfying

$$
\|u\|_{\mathscr{D}}^{2} \equiv \int_{D}\left|u^{\prime}(z)\right|^{2} F\left(\log \frac{1}{|z|}\right) d A(z)<\infty .
$$

Clearly $\mathscr{D}$ is a Hilbert space with norm $\|\cdot\|_{\mathscr{D}}$. We define a composition operator $T$ on $\mathscr{D}$ by

$$
T u=u \circ B,
$$

where $B$ is our inner function from $\S 2$. Since $B(0)=0$, it is plausible that $T$ carries $\mathscr{D}$ boundedly into itself, and we will see in $\S 5$ that this is indeed the case. We write $\|T\|$ for the norm of $T$ as an operator from $\mathscr{D}$ to $\mathscr{D}$. Let us denote the $H^{\infty}$ norm of a bounded analytic function $u$ on $D$ by $\|u\|_{\infty}$. 
Lemma 3.2. Let $u \in H^{\infty}$ with $u(0)=1$. Then $1-u \in \mathscr{D}$ and

$$
\|1-u\|_{\mathscr{D}}^{2} \leq K\|u\|_{\infty}^{2}
$$

for some constant $K>0$ depending only on $F$.

Proof. Let us write $1-u=\sum_{1}^{\infty} a_{n} z^{n}$ for $z \in D$, so that

$$
\|1-u\|_{\mathscr{D}}^{2}=\int\left|(1-u)^{\prime}\right|^{2} F\left(\log \frac{1}{|z|}\right) d A=2 \pi \sum_{1}^{\infty} n^{2}\left|a_{n}\right|^{2} v_{n-1},
$$

where $v_{n}=\int_{0}^{1} r^{2 n+1} F\left(\log \frac{1}{r}\right) d r$. Since $F(t) / t$ is nondecreasing on $(0, \infty)$, we see that $F(t)=O(t)$ as $t \rightarrow 0$, which easily implies that $v_{n-1}=O\left(n^{-2}\right)$ as $n \rightarrow \infty$. Thus there is a constant $K>0$ independent of $u$ such that

$$
\|1-u\|_{\mathscr{D}}^{2} \leq K\left(1+\sum_{1}^{\infty}\left|a_{n}\right|^{2}\right)=K \int_{0}^{2 \pi}\left|u\left(e^{i \theta}\right)\right|^{2} \frac{d \theta}{2 \pi} \leq K\|u\|_{\infty}^{2} .
$$

Now we can put together the pieces and obtain our distance estimates.

Lemma 3.3 (First distance estimate). There exist positive constants $a$ and $\kappa$, depending only on $F$ and $w$, such that

$$
\inf _{p \in P^{2}(\mu)} \int\left|\chi_{D}-p\right|^{2} d \mu \leq a\left(\varepsilon+\kappa\|T\|^{2}\right)^{|\Delta| / 2 \pi} .
$$

Proof. The left side of our inequality here is of course dominated by the expression (3.1). We have already estimated the sum of the first two terms on the right side of (3.1), and can now estimate the 3rd term. Lemma 3.2 tells us that $1-h \in \mathscr{D}$ and clearly $1-g=T(1-h)$. We see from Lemmas 3.1 and 3.2, together with the inequality (3.2a) that

$$
\begin{aligned}
\int_{D}|1-g|^{2} G d A & \leq 6 \int_{D}\left|(1-g)^{\prime}\right|^{2} H d A \leq 6\|T(1-h)\|_{\mathscr{D}}^{2} \\
& \leq 6\|T\|^{2}\|1-h\|_{\mathscr{D}}^{2} \leq 6 K\|T\|^{2}\|h\|_{\infty}^{2} .
\end{aligned}
$$

Now consider the parameters $t$ and $M$ defining $h$. Since $h$ is outer,

$$
1=h(0)^{2}=\exp \left\{\frac{1}{2 \pi} \int_{\partial D} \log |h|^{2} d \theta\right\}=M^{s / 2 \pi} t^{1-s / 2 \pi}
$$

with $s=|\Delta|=|\Gamma|$, whence $M=\left(\frac{1}{t}\right)^{2 \pi / s-1}$. It follows that (3.1) is bounded above by

$$
\varepsilon\left(\frac{1}{t}\right)^{2 \pi / s-1}+t \int w d \theta+6 K\|T\|^{2}\|h\|_{\infty}^{2}
$$

for any choice of $t>0$. Let us choose $t=\left(\varepsilon+6 K\|T\|^{2}\right)^{s / 2 \pi}$; if $t \leq 1$ (this is the useful case), we see that $\|h\|_{\infty}^{2}=M$ and we easily calculate (with this choice of $t$ ) that (3.3) is dominated by

$$
\left(1+\int w d \theta\right)\left(\varepsilon+6 K\|T\|^{2}\right)^{s / 2 \pi}
$$

and the conclusion follows. If $t>1$, the conclusion is trivial. 
Lemma 3.3 will be used in the proof of Theorems A and B; for Theorem C we need a slight variation.

Lemma 3.4 (Second distance estimate). There exists positive constants $a$ and $\kappa$, depending only on $F$ and $w$, such that

$$
\inf _{p \in P^{2}(\mu)} \int\left|\chi_{D}-p\right|^{2} d \mu \leq a \varepsilon^{|\Delta| / 2 \pi}+\kappa\|T\|^{2}\|1-h\|_{\mathscr{D}}^{2},
$$

where $h$ is the outer function described above with the choice $t=\varepsilon^{|\Delta| / 2 \pi}$. Proof. From our calculations above we know that (3.1) is dominated by

$$
\varepsilon\left(\frac{1}{t}\right)^{2 \pi / s-1}+t \int w d \theta+6 K\|T\|^{2}\|1-h\|_{\mathscr{D}}^{2}
$$

for any choice of $t>0$. The conclusion follows on taking $t=\varepsilon^{s / 2 \pi}$.

\section{AN ESTIMATE ON $B$}

The goal of this section is to show that for the inner function $B$ defined in $\S 2$, we have $|B(z)| \leq|z|^{\log n}$ on the annulus $R=\left\{z: \frac{1}{2}<|z|<1\right\}$. Recall that $B$ was constructed in the form $B=\lambda \circ \Psi$, where $\Psi$ is a Cayley inner function and

$$
\lambda(\zeta)=\frac{\zeta-e^{i s / 2}}{\zeta-e^{-i s / 2}}
$$

with $s=|\Delta|$. Recall that $B(0)=0$ and, in the notation of $\S 2, B$ maps almost all of the set $\Delta$ onto $\Gamma=\left\{e^{i x}: 0<x<s\right\}$. The inequality on $|B(z)|$ given above will be obtained by comparison of the Cayley inner function associated to $B$ with the Cayley inner function associated to the function $\alpha z^{n}$ on $D$. To this end, let $J_{1}, J_{2}, \ldots, J_{n}$ be equally spaced arcs of length $s / n$ in $\partial D$ with $J_{k}$ centered in $I_{k}$. ( $I_{k}$ has the same meaning here as in $\S 2$ : equally spaced arcs of length $1 / n$ used in the construction of $B$.) Set $J=\bigcup_{k=1}^{n} J_{k}$ and define the Cayley inner function

$$
\Phi(z)=\exp \left\{\frac{i}{2} \int_{J} \frac{e^{i \theta}+z}{e^{i \theta}-z} d \theta\right\} .
$$

Lemma 4.1. For some complex $\alpha$ with $|\alpha|=1, \lambda(\Phi(z))=\alpha z^{n}$.

Proof. Choose $u$ with $|u|=1$ so that the endpoints of $u J_{k}=\left\{u z: z \in J_{k}\right\}$ are $e^{i r_{k}}$ and $e^{i s_{k}}$ with $0 \leq r_{k}<s_{k}<2 \pi$ and $0=r_{1}<\cdots<r_{n}$. Then one computes

$$
\lambda^{-1}\left(u^{n} z^{n}\right)=e^{-i s / 2} \frac{u^{n} z^{n}-e^{i s}}{u^{n} z^{n}-1}=e^{-i s / 2} \prod_{k=0}^{n-1} \frac{u z-e^{i s / n} \omega_{k}}{u z-\omega_{k}},
$$

where $\omega_{k}=e^{2 \pi i k / n}$. On the other hand, a change of variables gives

$$
\Phi(z)=\prod_{k=1}^{n} \exp \left\{\frac{i}{2} \int_{u J_{k}} \frac{e^{i \theta}+u z}{e^{i \theta}-u z} d \theta\right\} .
$$


One can compute directly $[R R$, p. 296] that

$$
\exp \left\{\frac{i}{2} \int_{u J_{k}} \frac{e^{i \theta}+\zeta}{e^{i \theta}-\zeta} d \theta\right\}=e^{i\left(r_{k}-s_{k}\right) / 2} \frac{\zeta-e^{i s_{k}}}{\zeta-e^{i r_{k}}} .
$$

But in our setup, $r_{k}-s_{k}=-s / n, e^{i r_{k}}=\omega_{k-1}$ and $e^{i s_{k}}=e^{i s / n} \omega_{k-1}$, whence

$$
\Phi(z)=e^{-i s / 2} \prod_{k=1}^{n} \frac{u z-e^{i s / n} \omega_{k-1}}{u z-\omega_{k-1}}=\lambda^{-1}\left(u^{n} z^{n}\right) .
$$

The conclusion follows with $\alpha=u^{n}$.

In the next lemma we compare $\log \Psi$ and $\log \Phi$ on $|z|=\frac{1}{2}$.

Lemma 4.2. If $|z|=\frac{1}{2}$, then

$$
|\log \Psi(z)-\log \Phi(z)| \leq 2 \sqrt{2} \frac{s}{n} .
$$

Proof. By definition,

$$
\begin{aligned}
|\log \Psi(z)-\log \Phi(z)| & =\left|\frac{i}{2} \int_{\Delta} \frac{e^{i \theta}+z}{e^{i \theta}-z} d \theta-\frac{i}{2} \int_{J} \frac{e^{i \theta}+z}{e^{i \theta}-z} d \theta\right| \\
& \leq \sum_{k=1}^{n}\left|\frac{i}{2} \int\left(\chi_{\Delta_{k}}\left(e^{i \theta}\right)-\chi_{J_{k}}\left(e^{i \theta}\right)\right) \frac{e^{i \theta}+z}{e^{i \theta}-z} d \theta\right|
\end{aligned}
$$

where $\Delta_{k}=\Delta \cap I_{k}$ and $J_{k}=J \cap I_{k}$. For fixed $z$ with $|z|=\frac{1}{2}$, we write

$$
\frac{i}{2} \frac{e^{i \theta}+z}{e^{i \theta}-z}=U\left(e^{i \theta}\right)+i V\left(e^{i \theta}\right)
$$

with $U$ and $V$ real-valued. Let $m_{k}$ and $M_{k}$ denote the infimum and supremum, respectively, of $U$ over $I_{k}$. An easy calculation shows that $M_{k}-m_{k} \leq$ $2\left|I_{k}\right|$. Moreover, since $\left|J_{k}\right|=\left|\Delta_{k}\right|=s / n$ we have

$$
m_{k} \frac{s}{n} \leq \int \chi_{\Delta_{k}} U d \theta \leq M_{k} \frac{s}{n}
$$

and similarly with $\chi_{J_{k}}$ replacing $\chi_{\Delta_{k}}$. It follows that

$$
\left|\int\left(\chi_{\Delta_{k}}-\chi_{J_{k}}\right) U d \theta\right| \leq\left(M_{k}-m_{k}\right) \frac{s}{n} \leq 2\left|I_{k}\right| \frac{s}{n}=2 \frac{s}{n^{2}} .
$$

The same estimate holds with $V$ replacing $U$ and we conclude

$$
|\log \Psi(z)-\log \Phi(z)| \leq 2 \sqrt{2} \frac{s}{n^{2}} \cdot n=2 \sqrt{2} \frac{s}{n} .
$$

We now estimate $B(z)=\lambda(\Psi(z))$ for $|z|=\frac{1}{2}$.

Lemma 4.3. There is a universal constant $n_{0}$ so that if $n \geq n_{0}$,

$$
|B(z)| \leq \frac{14}{n} \quad \text { for }|z|=\frac{1}{2} \text {. }
$$


Proof. Our first task is to obtain the estimate

$$
\left|\log \Psi(z)-\frac{i s}{2}\right| \leq \frac{3 s}{n} \quad \text { if }|z|=\frac{1}{2}
$$

and $n \geq n_{0}$; we take the principal branch of the logarithm, $\log 1=0$. To this end write

$$
\left|\log \Psi(z)-\frac{i s}{2}\right| \leq|\log \Psi(z)-\log \Phi(z)|+\left|\log \Phi(z)-\frac{i s}{2}\right| .
$$

Now the first term has been shown in Lemma 4.2 not to exceed $2 \sqrt{2} \frac{s}{n}$. To estimate the second term we use Lemma 4.1 and the definition of $\lambda$ to write

$$
\left|\log \Phi(z)-\frac{i s}{2}\right|=\left|\log \lambda^{-1}\left(\alpha z^{n}\right)-\log \lambda^{-1}(0)\right| \text {. }
$$

By computing the derivative of $\log \lambda^{-1}(\zeta)$ and estimating its size for $|\zeta| \leq 2^{-n}$, one obtains the estimate

$$
\left|\log \lambda^{-1}\left(\alpha z^{n}\right)-\log \lambda^{-1}(0)\right| \leq \frac{s}{2^{n}\left(1-2^{-n}\right)^{2}}
$$

for $|z|=\frac{1}{2}$. Putting this together with the first part yields the desired result (4.1), provided $n \geq n_{0}$ and $n_{0}$ is sufficiently large.

Next we use (4.1) to obtain the desired inequality on $|B(z)|$ for $|z|=\frac{1}{2}$. Note that $B(z)=\tau(\log \Psi(z))$, where $\tau(z)=\lambda\left(e^{z}\right)$, so that

$$
|B(z)|=|\tau(\log \Psi(z))-\tau(\log \Psi(0))| \text {. }
$$

Let us denote the closed disk centered at $i s / 2$ and of radius $3 s / n$ by $W$. Clearly we need to estimate the size of $\tau^{\prime}$ on $W$. Now $\tau^{\prime}(z)=\lambda^{\prime}\left(e^{z}\right) e^{z}$ and an easy estimate shows that $\left|\lambda^{\prime}\right| \leq 2 / \sin (s / 2) \leq 9 /(2 s)$ in the upper half-plane (using $s \leq 1$ ). Thus for $z$ in $W$ and $n \geq n_{0}$, where $n_{0}$ is large enough,

$$
\left|\tau^{\prime}(z)\right| \leq \frac{9}{2 s} e^{3 s / n} \leq \frac{14}{3 s}
$$

Hence

as claimed.

$$
|B(z)|=|\tau(\log \Psi(z))-\tau(\log \Psi(0))| \leq \frac{14}{3 s} \cdot \frac{3 s}{n}=\frac{14}{n},
$$

Finally, we use Lemma 4.3 to obtain our desired estimate on $B$. We may take the universal constant here to be the same as in Lemma 4.3; just make it bigger if necessary.

Lemma 4.4. There is a universal constant $n_{0}$ such that

$$
|B(z)| \leq|z|^{\log n} \quad \text { for } \frac{1}{2}<|z|<1
$$

provided $n \geq n_{0}$.

Proof. Let $M(r)$ denote the maximum value of $|B(z)|$ on $|z|=r$. We have just shown in Lemma 4.3 that $M\left(\frac{1}{2}\right) \leq 14 / n$ provided $n \geq n_{0}$. For $r_{1}<1$ and 
arbitrarily close to 1 we have trivially $M\left(r_{1}\right) \leq 1$. Hence for $\frac{1}{2}<r<r_{1}<1$, Hadamard's Three Circles Theorem implies

$$
M(r)^{\log \left(2 r_{1}\right)} \leq\left(\frac{14}{n}\right)^{\log \left(r_{1} / r\right)} .
$$

We may let $r_{1} \rightarrow 1$ to find that

$$
M(r) \leq\left(\frac{14}{n}\right)^{\log (1 / r) / \log 2}<r^{\log n},
$$

where the last inequality holds provided $n>e^{9}$.

\section{COMPOSITION OPERATORS ON $\mathscr{D}$}

In this section we give a general method for estimating the norm of a composition operator, induced by an analytic map $\phi: D \rightarrow D$ with $\phi(0)=0$, and acting on a space $\mathscr{D}$ as defined in $\S 3$. We then apply this result to estimate $\|T\|$, where $T$ is the composition operator induced by the inner function $B$ of $\S 2$.

Before stating the main result of this section we record a change of variables theorem which will prove useful. Shapiro gives a version of this in [Sh]; the proof there, with obvious modifications, also yields the slightly more general formula we need. For each $w$ in $\phi(D)$, the range of $\phi$, we let $\left\{z_{j}(w)\right\}_{j \geq 1}$ denote the set of distinct zeros of $\phi-w$.

Lemma 5.1. Let $g$ and $E$ be nonnegative measurable functions on $D$. Then

$$
\int_{D} g(\phi(z))\left|\phi^{\prime}(z)\right|^{2} E(z) d A(z)=\int_{\phi(D)} g(w)\left(\sum_{j \geq 1} E\left(z_{j}(w)\right)\right) d A(w) .
$$

Since we are assuming $\phi(0)=0$ we also have Littlewood's inequality

$$
\sum_{j \geq 1} \log \frac{1}{\left|z_{j}(w)\right|} \leq \log \frac{1}{|w|}, \quad w \in \phi(D),
$$

which can be found in [Ne, p. 52] or readily deduced by evaluating both the function $(\phi-w)(1-\bar{w} \phi)^{-1}$ and its Blaschke factor at the origin.

For $0 \leq \rho<1$, let $R$ denote the annulus $\{z: \rho<|z|<1\}$. We omit the easy proof of the next lemma.

Lemma 5.2. There exists $c>0$, depending only on $\rho$ and $F$, such that

$$
\|u\|_{\mathscr{D}}^{2} \leq c \int_{R}\left|u^{\prime}\right|^{2} F\left(\log \frac{1}{|z|}\right) d A
$$

for every $u$ analytic on $D$ with $u(0)=0$. (Here we understand that such a $u$ lies in $\mathscr{D}$ exactly when $\|u\|_{\mathscr{D}}<\infty$.)

In the statement of the next theorem, which is the main result of this section, $T_{\phi}$ is the composition operator defined for $u \in \mathscr{D}$ by $T_{\phi} u=u \circ \phi$, and $c$ denotes the constant of Lemma 5.2. 
Theorem 5.3. Let $\phi: D \rightarrow D$ be analytic with $\phi(0)=0$. If there exist numbers $A>0$ and $0 \leq \rho<1$ so that $|\phi(z)| \leq|z|^{A}$ for $z$ in $R=\{\rho<|z|<1\}$, then $T_{\phi}$ is a bounded operator on $\mathscr{D}$ and

$$
\left\|T_{\phi}\right\|^{2} \leq c \sup _{t>0} \frac{A F(t / A)}{F(t)} .
$$

Before giving the proof of this theorem, we note a corollary which is our immediate goal. Recall that $T$ denotes the composition operator induced by the inner function $B$ defined in $\S 2$, and that $B$ depends (among other things) on an integer $n \geq 2$. In the following we assume $n \geq n_{0}$, where $n_{0}$ is the universal constant of Lemma 4.4.

Corollary 5.4. If $T$ is defined on $\mathscr{D}$ by $T u=u \circ B$, then $T$ is a bounded operator on $\mathscr{D}$ and

$$
\|T\|^{2} \leq c \sup _{t>0} \frac{\log n F(t / \log n)}{F(t)},
$$

where $c$ is the constant of Lemma 5.2 for $\rho=\frac{1}{2}$.

Proof. By Lemma 4.4, $|B(z)| \leq|z|^{\log n}$ on $\left\{z: \frac{1}{2}<|z|<1\right\}$. The result now follows immediately from Theorem 5.3.

Proof of Theorem 5.3. If $u \in \mathscr{D}$, Lemma 5.2 implies that

$$
\begin{aligned}
\left\|T_{\phi} u\right\|_{\mathscr{D}}^{2} & \leq c \int_{R}\left|\left(T_{\phi} u\right)^{\prime}\right|^{2} F\left(\log \frac{1}{|z|}\right) d A \\
& =c \int_{R}\left|u^{\prime}(\phi(z))\right|^{2}\left|\phi^{\prime}(z)\right|^{2} F\left(\log \frac{1}{|z|}\right) d A(z) .
\end{aligned}
$$

Now apply Lemma 5.1 with

$$
E(z)=\chi_{R}(z) F\left(\log \frac{1}{|z|}\right)
$$

to conclude that the right side of $(5.2)$ is equal to

$$
c \int_{\phi(D)}\left|u^{\prime}(w)\right|^{2}\left\{\sum_{j \geq 1} \chi_{R}\left(z_{j}(w)\right) F\left(\log \frac{1}{\left|z_{j}(w)\right|}\right)\right\} d A(w) .
$$

Now let $P(t)=F(t) / t$, a nondecreasing function on $(0, \infty)$. If $w \in \phi(D)$ and $\chi_{R}\left(z_{j}(w)\right) \neq 0$, we see that the hypothesis $|\phi(z)| \leq|z|^{A}$ for $z \in R$ yields

$$
\log \frac{1}{\left|z_{j}(w)\right|} \leq \frac{1}{A} \log \frac{1}{|w|}
$$

and hence

$$
P\left(\log \frac{1}{\left|z_{j}(w)\right|}\right) \leq P\left(\frac{1}{A} \log \frac{1}{|w|}\right)
$$


Thus for any $w \in \phi(D)$ we have, using the inequality (5.1),

$$
\begin{aligned}
\sum_{j \geq 1} \chi_{R}\left(z_{j}(w)\right) F\left(\log \frac{1}{\left|z_{j}(w)\right|}\right) & =\sum_{j \geq 1} \chi_{R}\left(z_{j}(w)\right) P\left(\log \frac{1}{\left|z_{j}(w)\right|}\right) \log \frac{1}{\left|z_{j}(w)\right|} \\
& \leq P\left(\frac{1}{A} \log \frac{1}{|w|}\right) \sum_{j \geq 1} \log \frac{1}{\left|z_{j}(w)\right|} \\
& \leq P\left(\frac{1}{A} \log \frac{1}{|w|}\right) \log \frac{1}{|w|}=A F\left(\frac{1}{A} \log \frac{1}{|w|}\right) .
\end{aligned}
$$

Thus (5.3) cannot exceed

$$
c \sup _{t>0} \frac{A F(t / A)}{F(t)} \int_{D}\left|u^{\prime}(w)\right|^{2} F\left(\log \frac{1}{|w|}\right) d A(w)=c \sup _{t>0} \frac{A F(t / A)}{F(t)}\|u\|_{\mathscr{D}}^{2}
$$

as desired.

We remark that since we always have $|\phi(z)| \leq|z|$ in $D$ by the Schwarz lemma, we may take $\rho=0, A=1$ and $c=1$ in Theorem 5.3 to conclude that $T_{\phi}$ is always a contraction operator on $\mathscr{D}$, with no hypothesis on $\phi$ besides $\phi(0)=0$.

It is interesting to apply Theorem 5.3 to estimate the norm of a composition operator acting on the subspace of a weighted Bergman space consisting of those functions which are zero at the origin. That is, for fixed $\alpha>-1$ we consider the weighted Bergman space $P^{2}\left((1-|z|)^{\alpha} d A\right)$ and let

$$
\mathscr{M}=\left\{u \in P^{2}\left((1-|z|)^{\alpha} d A\right): u(0)=0\right\} .
$$

From Lemma 3.1 and the fact that $1-r$ and $\log \frac{1}{r}$ are comparable for $r$ near 1 we conclude that the expression

$$
\left\{\int_{D}\left|u^{\prime}(z)\right|^{2}\left(\log \frac{1}{|z|}\right)^{\alpha+2} d A(z)\right\}^{1 / 2}
$$

gives an equivalent norm on $\mathscr{M}$. In other words, the space $\mathscr{D}$ associated to $F(t)=t^{\alpha+2}$ is exactly our subspace $\mathscr{M}$ with an equivalent norm. If $\phi: D \rightarrow D$ is analytic and $\phi(0)=0$, let us write $T_{\phi} \mid \mathscr{M}$ for $T_{\phi}$ considered as a bounded operator on $\mathscr{M}$. Theorem 5.3 immediately implies the following estimate.

Corollary 5.5. If $\phi: D \rightarrow D$ is analytic with $\phi(0)=0$, and if $|\phi(z)| \leq|z|^{A}$ on the annulus $\{z: \rho<|z|<1\}$, then

$$
\left\|T_{\phi} \mid \mathscr{M}\right\| \leq c A^{-(1+\alpha)}
$$

for some constant $c$ depending only on $\rho$ and $\alpha$.

This result contrasts sharply with the situation for composition operators acting on the Hardy space $H^{2}$ where, for example, if $\phi(0)=0$ and $\phi$ is inner, then $T_{\phi}$ is an isometry.

We may also relate the estimate for $\left\|T_{\phi} \mid \mathscr{M}\right\|$ to the location of the zeros of $\phi$. 
Proposition 5.6. Suppose $\phi: D \rightarrow D$ with $\phi(0)=0$, and let $\left\{\beta_{k}\right\}_{k \geq 1}$ be the zeros of $\phi$, repeated according to multiplicity. Then there is a constant $b>0$, depending only on $\alpha$, such that

$$
\left\|T_{\phi} \mid \mathscr{M}\right\|^{2} \leq b\left(\sum_{k \geq 1}\left(1-\left|\beta_{k}\right|\right)\right)^{-(1+\alpha)} .
$$

Proof. By Corollary 5.5 it suffices to show that $|\phi(z)| \leq|z|^{A}$ on the annulus $R=\left\{z: \frac{1}{2}<|z|<1\right\}$, where $A=a \sum_{k \geq 1}\left(1-\left|\beta_{k}\right|\right)$ and $a$ is a positive constant. The Blaschke factor $\left(z-\beta_{k}\right) /\left(1-\bar{\beta}_{k} z\right)$ of $\phi$ has modulus equal to

$$
\exp \left(\frac{1}{2} \log \left|\frac{z-\beta_{k}}{1-\bar{\beta}_{k} z}\right|^{2}\right) \text {. }
$$

Since $-\log r \geq 1-r$ on $(0,1]$ this is bounded above by

$$
\exp \left\{\frac{1}{2}\left(\left|\frac{z-\beta_{k}}{1-\bar{\beta}_{k} z}\right|^{2}-1\right)\right\} \text {. }
$$

Using the identity

$$
1-\left|\frac{z-\beta_{k}}{1-\bar{\beta}_{k} z}\right|^{2}=\frac{\left(1-|z|^{2}\right)\left(1-\left|\beta_{k}\right|^{2}\right)}{\left|1-\bar{\beta}_{k} z\right|^{2}}
$$

one obtains

$$
\left|\frac{z-\beta_{k}}{1-\bar{\beta}_{k} z}\right| \leq \exp \left(-\frac{1}{8}(1-|z|)\left(1-\left|\beta_{k}\right|\right)\right) \text {. }
$$

If $z \in R$,

$$
1-|z| \geq \frac{1}{2 \log 2} \log \frac{1}{|z|},
$$

and hence

$$
\left|\frac{z-\beta_{k}}{1-\bar{\beta}_{k} z}\right| \leq|z|^{a\left(1-\left|\beta_{k}\right|\right)}
$$

where $a=(16 \log 2)^{-1}$.

Thus for $z \in R$ we have

$$
|\phi(z)| \leq \prod\left|\frac{z-\beta_{k}}{1-\overline{\beta_{k}} z}\right| \leq|z|^{a \sum\left(1-\left|\beta_{k}\right|\right)}
$$

as desired.

\section{Above the log Cutoff: Theorems A And B}

In this section we consider the case where $G(r)$ decays to zero no more rapidly (roughly speaking) than $\exp (-C /(1-r))$. We present a general theorem from which Theorems A and B will be deduced. Let us begin with a simple lemma. 
Lemma 6.1. If $\delta_{1}<\delta_{2}$, then $\Omega\left(\delta_{1}, \varepsilon\right) \leq 2 \Omega\left(\delta_{2}, \varepsilon\right)$.

Proof. Let $I$ be an arbitrary arc of length $\delta_{2}$. Clearly it suffices to show that for $\varepsilon>0, \Omega_{I}(\varepsilon) \geq \frac{1}{2} \Omega\left(\delta_{1}, \varepsilon\right)$. Since $\delta_{1}<\delta_{2}$, we may write $I=L \cup\left(\bigcup_{k=1}^{n} J_{k}\right)$, where $L$ and each $J_{k}$ are arcs with $\left|J_{k}\right|=\delta_{1},|L| \leq \delta_{1}$, and $n \geq 1$. Now

$$
\begin{aligned}
\left|\left\{e^{i x} \in I: w\left(e^{i x}\right) \leq \varepsilon\right\}\right| & \geq \sum_{k=1}^{n}\left|\left\{e^{i x} \in J_{k}: w\left(e^{i x}\right) \leq \varepsilon\right\}\right| \\
& \geq \sum_{k=1}^{n} \Omega\left(\delta_{1}, \varepsilon\right)\left|J_{k}\right|=n \delta_{1} \Omega\left(\delta_{1}, \varepsilon\right) .
\end{aligned}
$$

Thus

$$
\Omega_{I}(\varepsilon) \geq \frac{1}{|I|} n \delta_{1} \Omega\left(\delta_{1}, \varepsilon\right) \geq \frac{1}{2} \Omega\left(\delta_{1}, \varepsilon\right) .
$$

We can now state and prove the main theorem of this section. Recall $\mu=$ $G d A+w d \theta$ and $F$ is any positive measurable function on $(0, \infty)$ satisfying

$$
\begin{aligned}
& \int_{r}^{1}\left(\int_{x}^{1} G(y) d y\right) d x \leq F\left(\log \frac{1}{r}\right), \quad 0<r<1, \\
& \int_{0}^{\infty} F(t) e^{-2 t} d t<\infty \\
& F(t) / t \text { is nondecreasing on }(0, \infty) .
\end{aligned}
$$

If $n \geq 2$, let

$$
\beta_{n}=\sup _{t>0} \frac{\log n F(t / \log n)}{F(t)}
$$

Theorem 6.2. Suppose $t_{0}>0$ and let $f:\left(0, t_{0}\right) \rightarrow(0,2 \pi)$ be a strictly increasing continuous function with $f(t) \rightarrow 0$ as $t \rightarrow 0$. Suppose that for some sufficiently large $N>0$ and all integers $n \geq N$ the numbers $\varepsilon_{n}$ defined by $f\left(\varepsilon_{n}\right)=\frac{1}{n}$ satisfy $\varepsilon_{n+1} \geq \varepsilon_{n}^{K}$ and $\varepsilon_{n}>\beta_{n}^{d}$ for some positive constants $K$ and d. If

$$
\sup _{0<\varepsilon<t_{0}} \Omega(f(\varepsilon), \varepsilon) \log \frac{1}{\varepsilon}=+\infty
$$

then

$$
P^{2}(\mu)=P^{2}(G d A) \oplus L^{2}(w d \theta)
$$


Proof. Given $M>0$ we may by hypothesis choose $\varepsilon^{*}$ in $(0,1)$ such that

$$
\Omega\left(f\left(\varepsilon^{*}\right), \varepsilon^{*}\right) \log \frac{1}{\varepsilon^{*}}>M .
$$

Clearly the sequence $\left\{\varepsilon_{n}\right\}$ (defined in the statement of the theorem) decreases to 0 , so if $M$ is large enough, $\varepsilon^{*}$ will be close enough to 0 so that $\varepsilon_{n+1}<\varepsilon^{*} \leq \varepsilon_{n}$ for some $n \geq N$. Note that $\varepsilon_{n}^{K} \leq \varepsilon_{n+1}<\varepsilon^{*}$. We find

$$
\begin{aligned}
\Omega\left(\frac{1}{n}, \varepsilon_{n}\right) \log \frac{1}{\varepsilon_{n}} & \geq \Omega\left(\frac{1}{n}, \varepsilon^{*}\right) \log \frac{1}{\varepsilon_{n}} \geq \frac{1}{K} \Omega\left(\frac{1}{n}, \varepsilon^{*}\right) \log \frac{1}{\varepsilon^{*}} \\
& \geq \frac{1}{2 K} \Omega\left(f\left(\varepsilon^{*}\right), \varepsilon^{*}\right) \log \frac{1}{\varepsilon^{*}} \geq \frac{M}{2 K},
\end{aligned}
$$

where in the penultimate inequality we have used $f\left(\varepsilon^{*}\right) \leq \frac{1}{n}$ plus Lemma 6.1.

Let us associate a measurable set $\Delta \subset \partial D$ with $n$ and $\varepsilon=\varepsilon_{n}$ as follows. Select $\operatorname{arcs} I_{1}, I_{2}, \ldots, I_{n}$, equally spaced around $\partial D$ and each of length $\frac{1}{n}$. By the definition of $\Omega(\delta, \varepsilon)$ we can select, for each $k=1,2, \ldots, n$, a measurable subset $\Delta_{k} \subset I_{k}$ such that $w \leq \varepsilon_{n}$ on $\Delta_{k}$ and $\left|\Delta_{k}\right|=\Omega\left(\frac{1}{n}, \varepsilon_{n}\right) \frac{1}{n}$. We put $\Delta=\bigcup_{k=1}^{n} \Delta_{k}$ and note that $|\Delta|=\Omega\left(\frac{1}{n}, \varepsilon_{n}\right)$. Let $B$ and $T$ be, respectively, the inner function and composition operator associated to $\Delta$ as in $\S \S 2$ and 3. According to Corollary 5.4, $\|T\|^{2} \leq c \beta_{n}$ (we can clearly assume that $n \geq n_{0}$, simply by taking $M$ large enough). Also, without loss of generality we may take $d \geq 1$ in our hypothesis. By Lemma 3.3 and the above chain of inequalities

$$
\begin{aligned}
\inf _{p \in P^{2}(\mu)} \int\left|\chi_{D}-p\right|^{2} d \mu & \leq a\left(\varepsilon_{n}+\kappa\|T\|^{2}\right)^{\Omega\left(\frac{1}{n}, \varepsilon_{n}\right) / 2 \pi} \leq a\left(\varepsilon_{n}+\kappa c \beta_{n}\right)^{\Omega\left(\frac{1}{n}, \varepsilon_{n}\right) / 2 \pi} \\
& \leq a(1+\kappa c) \varepsilon_{n}^{\Omega\left(\frac{1}{n}, \varepsilon_{n}\right) / 2 \pi d} \leq a(1+\kappa c) e^{-M / 4 \pi K d} .
\end{aligned}
$$

Now let $M \rightarrow \infty$ to conclude that $\chi_{D} \in P^{2}(\mu)$.

We next interpret Theorem 6.2 using two natural choices for $G$. In both cases we determine the appropriate choices for $f$, depending on $\left\{\beta_{n}\right\}$, so as to satisfy the hypotheses of Theorem 6.2. The reader will note that an essential change, as we vary $G$, is the ratio of $f(\varepsilon)$ to $\varepsilon$.

Our first class of examples consists of the weights $G(r)=(1-r)^{\alpha}, \alpha>-1$. In this case the spaces $P^{2}(G d A)$ are the standard weighted Bergman spaces. One computes directly that

$$
\int_{r}^{1}\left[\int_{x}^{1} G(y) d y\right] d x=(\alpha+1)^{-1}(\alpha+2)^{-1}(1-r)^{\alpha+2} .
$$

Since $\log \frac{1}{r} \geq 1-r$, condition (a) above will be satisfied with

$$
F(t)=t^{\alpha+2} /(\alpha+1)(\alpha+2) \text {. }
$$

Since $\alpha>-1, F$ will also satisfy conditions (b) and (c). Moreover, $\beta_{n}=$ $(\log n)^{-1-\alpha}$. Setting $f(t)=e^{-1 / t^{7}}$ (where $\gamma>0$ ), or equivalently, $\varepsilon_{n}=$ $(\log n)^{-1 / \gamma}$ in Theorem 6.2 yields: 
Theorem A. Let $\mu=(1-|z|)^{\alpha} d A+w d \theta$ where $\alpha>-1$. Suppose $\gamma>0$ and let $f(t)=e^{-1 / t^{\prime}}$. If

$$
\sup _{0<\varepsilon<1} \Omega(f(\varepsilon), \varepsilon) \log \frac{1}{\varepsilon}=+\infty,
$$

then $P^{2}(\mu)=P^{2}\left((1-|z|)^{\alpha} d A\right) \oplus L^{2}(w d \theta)$.

We next consider functions $G$ which decay exponentially.

Theorem B. Let $\mu=G d A+w d \theta$, where $G(r)=\exp \left(-(1 /(1-r))^{\alpha}\right), 0<\alpha \leq$ 1. Set $f(t)=\exp \left(-\left(\log \frac{1}{t}\right)^{(\alpha+1) / \alpha}\right)$. If

$$
\sup _{0<\varepsilon<1} \Omega(f(\varepsilon), \varepsilon) \log \frac{1}{\varepsilon}=+\infty,
$$

then $P^{2}(\mu)=P^{2}(G d A) \oplus L^{2}(w d \theta)$.

Proof. We begin by defining a function $F(t)$ satisfying (a), (b), and (c) above. One shows easily that $F_{0}(t)=\exp \left(-t^{-\alpha}+t\right)$ satisfies $(\mathrm{a})$ and $(\mathrm{b})$, but that $F_{0}(t) / t$ may be decreasing on an interval $\left(t_{1}, t_{2}\right)$ with $0<t_{1}<t_{2}<1$. Modify this choice by setting $F(t)=F_{0}(t) Q(t)$ where $Q(t)$ is a nondecreasing function which is 1 on $\left(0, t_{1}\right)$, increasing sufficiently rapidly on $\left(t_{1}, t_{2}\right)$ so that $F(t) / t$ will be nondecreasing there, and constant on $\left(t_{2}, \infty\right)$; clearly conditions (a) and (b) are preserved under this modification. Moreover, if $n \geq 3$,

$$
\beta_{n} \leq \sup _{t>0} \frac{\log n F_{0}(t / \log n)}{F_{0}(t)}
$$

since $Q$ is nondecreasing. A computation shows that

$$
\sup _{t>0} \frac{F_{0}(t / \log n)}{F_{0}(t)} \leq \exp \left(-c(\alpha)(\log n)^{\alpha /(\alpha+1)}\right),
$$

where $c(\alpha)$ is a positive constant depending only on $\alpha$. If

$$
f(t)=\exp \left(-(\log (1 / t))^{(\alpha+1) / \alpha}\right) \text { and } \varepsilon_{n}=\exp \left(-(\log n)^{\alpha /(\alpha+1)}\right),
$$

then $f\left(\varepsilon_{n}\right)=\frac{1}{n}$ and $\varepsilon_{n} \geq \beta_{n}^{2 / c(\alpha)}$ for large $n$. Thus the theorem follows from Theorem 6.2.

In comparing Theorems $\mathrm{A}$ and $\mathrm{B}$ one expects to find that the hypotheses become weaker as the weight function $G(r)$ decreases more rapidly as $r \uparrow 1$. This is indeed the case, as can be seen by first noting that the function $f$ tends to zero more rapidly in Theorem A and less rapidly in Theorem B. Moreover, Lemma 6.1 implies that the hypothesis

$$
\sup _{0<\varepsilon<t_{0}} \Omega(f(\varepsilon), \varepsilon) \log \frac{1}{\varepsilon}=+\infty
$$

becomes easier to satisfy as $f$ tends to zero more slowly. 
We complete this section with a construction of weight functions $w$ for which $\Omega(\delta, \varepsilon)$ will have prescribed behavior. Recall that $\Omega(\delta, \varepsilon)$ typically tends to zero as $\delta$ and $\varepsilon$ individually tend to zero. The content of the next proposition is that within this constraint one may construct positive functions $w$ on $\partial D$ for which $\Omega(\delta, \varepsilon)$ decreases as slowly as desired. Hence there is no difficulty in finding functions $w$ to satisfy the hypotheses of Theorem 6.2.

Proposition 6.3. Let $p$ and $q$ be strictly increasing continuous functions mapping $[0,1]$ onto $[0,1]$ (so that $p(0)=q(0)=0)$. Then there exists a positive bounded measurable function $w$ on $\partial D$ and a positive number $C$ for which

$$
\Omega(\delta, \varepsilon) \geq C q(\delta) p(\varepsilon), \quad 0<\delta, \varepsilon<1 .
$$

Proof. It clearly suffices to construct $w$ with $w>0$ a.e. For notational convenience, we define $w$ on $[0,1]$ instead of $\partial D$. For $a, x \in[0,1]$ (with $a>0$ ) and fixed $r \in(0,1)$, let

$$
h(a, x)= \begin{cases}x^{-r} & \text { if } 0<x \leq a, \\ a^{-r} & \text { if } x=0 \\ 0 & \text { otherwise }\end{cases}
$$

We call any translate of $h$ a spike of width $a$. For $n=1,2,3, \ldots$, choose positive numbers $i(n) \uparrow \infty$ as $n \uparrow \infty$ so that $q(1 / i(n))^{r}=2^{-n}$. We define $g_{n}(x)$ on $[0,1]$ as follows. On $[0,1 / i(n)], g_{n}(x)=h(1 / i(n), x)$. Let $[i(n)]$ denote the greatest integer in $i(n)$. Extend $g_{n}$ to all of $[0,[i(n)] / i(n)]$ to be periodic of period $1 / i(n)$. Finally, if $i(n)$ is not an integer, define $g_{n}$ on $([i(n)] / i(n), 1]$ by

$$
g_{n}(x)=h\left(\frac{1}{i(n)}, x-\frac{[i(n)]}{i(n)}\right) .
$$

Thus $g_{n}$ consists of $[i(n)]$ "full spikes" of width $1 / i(n)$, together with (possibly) a final spike of width less than $1 / i(n)$. Note that

$$
\int_{0}^{1} g_{n}(x) d x \leq \frac{i(n)^{r-1}}{1-r}([i(n)]+1) \leq \frac{2}{1-r} i(n)^{r} .
$$

Next, set $a_{n}=2^{-n} i(n)^{-r}$ and consider $g=\sum_{n=1}^{\infty} a_{n} g_{n}$. The above inequality shows that $g \in L^{1}[0,1]$ and hence that $g$ is finite a.e.

Next we define a strictly increasing function $\phi$ on $(0, \infty)$ by $\phi\left(\log \frac{1}{\varepsilon}\right)=$ $p(\varepsilon)^{-r}$ and set $w(x)=\exp \left(-\phi^{-1}(g(x))\right)$. Since $g<\infty$ a.e., $w$ will be a.e. positive. To complete the proof of the proposition, we need to estimate the measure of the sets $F_{\varepsilon} \equiv\{x \in I: w(x) \leq \varepsilon\}$, where $I$ is an arc with $|I|=\delta$. By the definition of $w$,

$$
\left|F_{\varepsilon}\right|=\left|\left\{x: \chi_{I}(x) g(x) \geq \phi\left(\log \frac{1}{\varepsilon}\right)\right\}\right| .
$$

For $\delta$ less than some sufficiently small $\delta_{0}$, we may choose $N$ so that $1 / i(N) \leq$ $\delta$ but $1 / i(N-1)>\delta$. Since $g=\sum a_{n} g_{n} \geq a_{N} g_{N}$, where the function $g_{N}$ is as defined above, using intervals of length $1 / i(N)$, we have

$$
\left|F_{\varepsilon}\right| \geq\left|\left\{x: \chi_{I} g_{N} \geq \phi\left(\log \frac{1}{\varepsilon}\right) / a_{N}\right\}\right| \text {. }
$$


By considering the graph of $g_{N}$ on $I$, one sees that

$$
\left|\left\{\chi_{I} g_{N} \geq \eta\right\}\right| \geq \min \left\{|I|,[\delta i(N)] \eta^{-1 / r}\right\} \geq \min \left\{\delta, 2^{-1} \delta i(N) \eta^{-1 / r}\right\} .
$$

Using this in (6.2), together with the definition of $a_{N}$, gives

$$
\left|F_{\varepsilon}\right| \geq \min \left\{\delta, 2^{-(N+r) / r} \delta p(\varepsilon)\right\} .
$$

Recall that $2^{-(N-1) / r}=q(1 / i(N-1))$ so that

$$
2^{-N / r}=2^{-1 / r} q\left(\frac{1}{i(N-1)}\right) \geq 2^{-1 / r} q(\delta)
$$

by our choice of $N$. Thus, for $\delta<\delta_{0}$

$$
\left|F_{\varepsilon}\right| \geq \min \left\{\delta, 2^{-(1+r) / r} \delta q(\delta) p(\varepsilon)\right\}=2^{-(1+r) / r} \delta q(\delta) p(\varepsilon),
$$

or equivalently, $\Omega(\delta, \varepsilon) \geq 2^{-(1+r) / r} q(\delta) p(\varepsilon)$ since $I$ is an arbitrary interval of length $\delta$. Finally, we may replace $2^{-(1+r) / r}$ by a sufficiently small positive constant $C$ so that $\Omega(\delta, \varepsilon) \geq C q(\delta) p(\varepsilon)$ holds for all $\delta<1$.

\section{Below the log Cutoff: Theorems C, $C^{\prime}$ and D}

In this section we consider weights $G$ which decay to zero faster than $\exp (-C /(1-r))$, and also present our necessary condition for splitting, Theorem $D$. The essential change here (as compared with the results of $\S 6$ ) is that splitting is guaranteed by the hypothesis

$$
\sup _{0<\varepsilon<t_{0}} \Omega(f(\varepsilon), \varepsilon) \log \frac{1}{\varepsilon}=+\infty
$$

for any choice of $f(t)$, provided $f(t)$ tends to zero with $t$. That such freedom in choosing $f$ is not available in the settings of Theorems $\mathrm{A}$ and $\mathrm{B}$ will be made clear in the next section.

Our most general result is formulated in terms of $\Omega_{I}(\varepsilon)$, the proportion of the arc $I$ on which $w \leq \varepsilon$. Recall that $\log w \notin$ weak $L^{1}(I)$ exactly when

$$
\sup _{0<\varepsilon<1} \Omega_{I}(\varepsilon) \log \frac{1}{\varepsilon}=+\infty .
$$

Theorem C. Suppose that $\mu=G d A+w d \theta$ where $G(r)$ is decreasing for $r$ near 1 and satisfies

$$
\lim _{r \uparrow 1}(1-r) \log \frac{1}{G(r)}=+\infty .
$$

Suppose that for every pair $N_{1}, N_{2}$ of positive numbers there exists $\varepsilon>0$, an integer $n \geq N_{2}$, and equally spaced arcs $I_{1}, \ldots, I_{n}$ in $\partial D$, each of length $\frac{1}{n}$, such that

$$
\Omega_{I_{k}}(\varepsilon) \log \frac{1}{\varepsilon}>N_{1}, \quad k=1,2, \ldots, n .
$$

Then $P^{2}(\mu)=P^{2}(G d A) \oplus L^{2}(w d \theta)$. 
Examples of functions $G$ which satisfy the hypothesis of Theorem $C$ are the exponential weights (1.3) with $\alpha>1$ as well as the double exponential weights. We postpone the proof of Theorem $\mathrm{C}$ until the end of the section.

For the purpose of comparison with Theorems A and B we state an alternate version of this result.

Theorem $\mathbf{C}^{\prime}$. Suppose that $\mu=G d A+w d \theta$ where $G(r)$ is decreasing for $r$ near 1 and satisfies

$$
\lim _{r \uparrow 1}(1-r) \log \frac{1}{G(r)}=+\infty \text {. }
$$

If there exist sequences $\left\{\delta_{n}\right\}$ and $\left\{\varepsilon_{n}\right\}$ of positive numbers, both tending to zero, such that

$$
\lim _{n \rightarrow \infty} \Omega\left(\delta_{n}, \varepsilon_{n}\right) \log \frac{1}{\varepsilon_{n}}=+\infty,
$$

then $P^{2}(\mu)=P^{2}(G d A) \oplus L^{2}(w d \theta)$.

Proof. We derive Theorem $\mathrm{C}^{\prime}$ from Theorem C. Assume that the hypotheses of Theorem $\mathrm{C}^{\prime}$ hold. Let $N_{1}, N_{2}>0$, choose any integer $n \geq N_{2}$, and let $I_{1}, \ldots, I_{n}$ be equally spaced arcs in $\partial D$, each of length $\frac{1}{n}$. Choose $j$ such that $\delta_{j} \leq \frac{1}{n}$ and

$$
\Omega\left(\delta_{j}, \varepsilon_{j}\right) \log \frac{1}{\varepsilon_{j}}>2 N_{1} .
$$

Setting $\varepsilon=\varepsilon_{j}$ we have, for each $k=1,2, \ldots, n$ (using Lemma 6.1),

$$
\Omega_{I_{k}}(\varepsilon) \log \frac{1}{\varepsilon} \geq \Omega\left(\frac{1}{n}, \varepsilon\right) \log \frac{1}{\varepsilon} \geq \frac{1}{2} \Omega\left(\delta_{j}, \varepsilon\right) \log \frac{1}{\varepsilon}>N_{1} .
$$

The conclusion now follows from Theorem $\mathrm{C}$.

The next result is Theorem $\mathrm{D}$, our necessary condition for splitting. We require that $G$ satisfy a mild technical hypothesis, namely, that there exists $\rho$, $0<\rho<1$, so that $G$ is continuously differentiable on $(\rho, 1)$ and satisfies

$$
G(r)^{b} \leq-G^{\prime}(r) \leq G(r)^{-d}, \quad \rho<r<1,
$$

where $b$ and $d$ are positive constants with $b<2$.

Theorem D. Suppose that $\mu=G d A+w d \theta$ where $G$ satisfies (7.3) and $G(r) \rightarrow$ 0 as $r \rightarrow 1$. Suppose further that

$$
\int_{1-\delta}^{1} \log \log \frac{1}{G(r)} d r<\infty \quad(\delta \text { small }) .
$$

If $P^{2}(\mu)$ splits, then there is no arc $I \subset \partial D$ with $\log w \in L^{1}(I)$.

Note. The technical hypothesis (7.3) can sometimes be avoided. Consider, for example, any weight $G(r)$ which is bounded away from zero for $r$ near 1 . Then (7.3) may fail (and (7.4) may not even make sense), but Theorem $D$ applies to $\mu=G d A+w d \theta$ nevertheless. To see this, observe that $G(r) \geq 1-r$ for $r$ 
near 1 , so that if $P^{2}(\mu)$ splits, so does $P^{2}((1-|z|) d A+w d \theta)$; moreover the weight $1-r$ satisfies both (7.3) and (7.4).

The proof of Theorem $\mathrm{D}$ is a minor but important modification of the proof of Theorem 3 in [Kr1]. For completeness we sketch the argument. The following lemma is crucial; its proof is contained within the proof of Lemma 4 in [W].

Wermer's Lemma. Let $P$ be a positive, bounded measurable function on the real line $\mathbf{R}$ satisfying

$$
\int_{-\infty}^{\infty} \frac{\log P(x)}{1+x^{2}} d x>-\infty
$$

with $P(x) P(y) \leq P(x+y)$ for all $x, y$. If $I \subset \mathbf{R}$ is a nonempty open interval, then there exists a continuous function $h$ on $\mathbf{R}$, supported on $I$ but not identically zero, whose Fourier transform $\hat{h}$ satisfies $|\hat{h}(x)| \leq P(x)$ for all $x$.

Proof of Theorem D. Assume that $\mu$ satisfies the hypotheses. Since $P^{2}(\mu)$ splits, there is no harm in assuming that $w \leq 1$ a.e. and that

$$
\int_{0}^{1}\left(1-r^{2}\right)^{-1} G(r) r d r<\infty .
$$

We write

$$
P(x)=\int_{0}^{1} r^{2|x|+1} G(r) d r, \quad x \in \mathbf{R},
$$

so that in our notation from $\S 1, P(n)=p_{n}, n=0,1,2, \ldots$. Clearly we may assume that $P(0)<1$. An application of Hölder's inequality to the integral defining $P$ shows that $-\log P$ is concave down on $[0, \infty)$. Since $-\log P$ is also positive, it is easy to see that it is subadditive, that is $P(x) P(y) \leq P(x+y)$ for $x, y \geq 0$. This last inequality persists for all real $x, y$. Condition (7.3) allows us to invoke Theorem 7 of [Kr1] which states that convergence of the integral in (7.4) is equivalent to

$$
\int_{-\infty}^{\infty} \frac{\log P(x)}{1+x^{2}} d x>-\infty .
$$

Now assume, for the purpose of obtaining a contradiction, that there is a nontrivial arc $I \subset \partial D$ with $\log w \in L^{1}(I)$. Let us think of $\partial D$ as a real interval of length $2 \pi$, so that $I$ becomes an interval in $\mathbf{R}$. We have verified that $P$ satisfies the hypotheses of Wermer's lemma; let $h$ be the continuous function, supported on $I$ and with $|\hat{h}(x)| \leq P(x)$, which that lemma produces. On considering $h$ as defined on $\partial D$ by restriction, we see that its $n$th Fourier coefficient $c_{n}$ satisfies

$$
\left|c_{n}\right|=\frac{1}{2 \pi}|\hat{h}(n)|<P(n)=p_{n}
$$

for $n=0,1,2, \ldots$. 
Now let $a_{n}=-c_{n} / p_{n}$ and put $f(z)=\sum_{n=0}^{\infty} a_{n} z^{n}$. We check that $f$ lies in $P^{2}(G d A)$ :

$$
\begin{aligned}
\int|f|^{2} G d A & =2 \pi \sum_{n=0}^{\infty}\left|a_{n}\right|^{2} p_{n}=2 \pi \sum_{n=0}^{\infty} \frac{\left|c_{n}\right|^{2}}{p_{n}} \\
& <2 \pi \sum_{n=0}^{\infty} p_{n}=2 \pi \int_{0}^{1}\left(1-r^{2}\right)^{-1} G(r) r d r<\infty
\end{aligned}
$$

as desired.

Now, note that if $n \geq 0$,

$$
\int_{D} f \bar{z}^{n} G d A+\int_{\partial D} h \bar{z}^{n} d \theta=2 \pi a_{n} p_{n}+2 \pi c_{n}=0,
$$

so that the pair $f \oplus h$ is orthogonal in $P^{2}(G d A) \oplus L^{2}(d \theta)$ to the subspace spanned by $\left\{z^{n} \oplus z^{n}\right\}_{n=0}^{\infty}$; this subspace is of course $P^{2}(G d A+d \theta)$. Since $\log w \in L^{1}(I)$, there exists an outer function $g$ in $H^{\infty}$ with $|g|=w$ a.e. on $I$ and $|g|=1$ a.e. on $\partial D \backslash I$. Since $z^{n} g$ lies in $P^{2}(G d A+d \theta)$ when $n=0,1,2, \ldots$, we see that

$$
0=\int_{D} f \overline{z^{n} g} G d A+\int_{\partial D} h \overline{z^{n} g} d \theta=\int_{D}(f \bar{g}) \bar{z}^{n} G d A+\int_{I} h \frac{\bar{g}}{w} \bar{z}^{n} w d \theta
$$

for $n=0,1,2, \ldots$. Now consider the bounded function $v$ which agrees with $h \bar{g} / w$ on $I$ and vanishes on $\partial D \backslash I$. Further let $u$ denote the orthogonal projection of $f \bar{g}$ (an element of $L^{2}(G d A)$ ) onto $P^{2}(G d A)$. We see from the last equation that $u \oplus v$ is a nonzero vector in $P^{2}(G d A) \oplus L^{2}(w d \theta)$ which is orthogonal to $P^{2}(\mu)$, a contradiction of our hypothesis that $P^{2}(\mu)$ splits.

It is interesting to apply Theorems $\mathrm{C}^{\prime}$ and $\mathrm{D}$ to the special case of $w=\chi_{E}$. The resulting corollary also follows from Theorem 1.2 and Theorem D.

Corollary 7.1. Let $\mu=G d A+\chi_{E} d \theta$ where $G$ satisfies the technical hypothesis (7.3). Suppose that conditions (7.1) and (7.4) hold. Then $P^{2}(\mu)=P^{2}(G d A) \oplus$ $L^{2}(E)$ if and only if $|I \backslash E|>0$ for every arc $I \subset \partial D$.

Proof. For any arc $I \subset \partial D$ and $0<\varepsilon<1$ we have

$$
\Omega_{I}(\varepsilon)=|I \backslash E| /|I| \text {. }
$$

Suppose now that $|I \backslash E|>0$ for every arc $I$. Fix $\delta \in(0,2 \pi)$ and let $I(\theta)$ be the arc with center $e^{i \theta}$ and with $|I(\theta)|=\delta$. Note that $|I(\theta) \backslash E|$ is a positive continuous function of $\theta$ and thus

$$
\Omega(\delta, \varepsilon)=\inf _{\theta} \frac{|I(\theta) \backslash E|}{\delta}
$$

is positive as well as independent of $\varepsilon$. Given any sequence $\delta_{n} \downarrow 0$, we can thus choose $\varepsilon_{n} \downarrow 0$ with

$$
\lim _{n \rightarrow \infty} \Omega\left(\delta_{n}, \varepsilon_{n}\right) \log \frac{1}{\varepsilon_{n}}=+\infty
$$


and Theorem $\mathrm{C}^{\prime}$ implies that $P^{2}(\mu)$ splits. Conversely, if there exists an arc $I$ with $|I \backslash E|=0$, then $\log \chi_{E} \in L^{1}(I)$ and we may apply Theorem D to conclude that $P^{2}(\mu)$ does not split.

In order to prove Theorem $\mathrm{C}$ we will need to construct a function $F$ (and an associated weighted Dirichlet space $\mathscr{D}$ as described in $\S 3$ ); we want $F$ to satisfy the conditions $(3.2 \mathrm{a}),(3.2 \mathrm{~b})$, and $(3.2 \mathrm{c})$. Since we can alter $G(r)$ on any interval $[0, \rho], 0<\rho<1$, without affecting the conclusion of the theorem, we are free to assume that $G$ is bounded and decreasing on all of $[0,1)$. Now let $J(t)=G(1-t)$ for $0<t \leq 1$. We extend $J$ to be increasing and continuous on all of $(0, \infty)$, and define $F(t)=t^{2} J(t)$. Clearly we can assume that

$$
\int_{0}^{\infty} e^{-2 t} F(t) d t<\infty .
$$

Note also that $F(t) / t=t J(t)$ is nondecreasing. Since $G$ is decreasing and $F$ is increasing, we have

$$
\begin{aligned}
\int_{r}^{1}\left(\int_{x}^{1} G(s) d s\right) d x & \leq(1-r)^{2} G(r)=F(1-r) \\
& \leq F\left(\log \frac{1}{r}\right), \quad 0<r<1 .
\end{aligned}
$$

Thus $F$ satisfies conditions (3.2) and we define the space $\mathscr{D}$ as in $\S 3$ with norm given by

$$
\|u\|_{\mathscr{D}}^{2}=\int_{D}\left|u^{\prime}\right|^{2} F\left(\log \frac{1}{|z|}\right) d A .
$$

The proof of Theorem $\mathrm{C}$ will ultimately invoke the distance estimate Lemma 3.4; for now recall the outer function $h$ which appears in that estimate:

$$
|h|^{2}= \begin{cases}\varepsilon^{-1} \varepsilon^{s / 2 \pi} & \text { a.e. on } \Gamma, \\ \varepsilon^{s / 2 \pi} & \text { a.e. on } \partial D \backslash \Gamma,\end{cases}
$$

where $\Gamma=\left\{e^{i x}: 0<x<s\right\}$. We wish to estimate $\|1-h\|_{\mathscr{D}}$, a quantity which depends on the parameters $\varepsilon$ and $s$ and which we expect to be large. However, the hypotheses (7.1) will allow us to dominate $\|1-h\|_{\mathscr{D}}$ by a function of the single quantity $\varepsilon^{s}$, and this will be enough for our purposes. The resulting estimate is best expressed in terms of the strange function

$$
\Theta(t)=\left(\log \frac{1}{t}\right)^{2} \int_{D} \exp \left\{\frac{1}{\pi}\left(\log \frac{1}{t}\right) \frac{1}{1-|z|}\right\} \frac{J(a(1-|z|))}{(1-|z|)^{4}} d A(z),
$$

where $a=2 \log 2$.

Lemma 7.2. If (7.1) holds, then $\Theta(t)<\infty$ for all $t>0$.

Proof. Our hypothesis (7.1) on $G$ implies that if $K>0$, then

$$
J(a(1-|z|)) \leq \exp (-K /(1-|z|))
$$

provided $|z|$ is near enough to 1 . The finiteness of $\Theta(t)$ follows. 
Lemma 7.3. Suppose that (7.1) holds. Given $\varepsilon \in(0,1)$ and $s \in(0,1]$, let $h$ be the outer function defined by (7.5). Then

$$
\|1-h\|_{\mathscr{D}}^{2} \leq b \Theta\left(\varepsilon^{s}\right)
$$

where $b>0$ depends only on $F$.

Proof. Let $R=\left\{z: \frac{1}{2}<|z|<1\right\}$. We have

$$
\log \frac{1}{|z|} \leq a(1-|z|), \quad z \in R,
$$

where $a=2 \log 2$. Since $f$ is nondecreasing,

$$
F\left(\log \frac{1}{|z|}\right) \leq F(a(1-|z|)) \leq a^{2} J(a(1-|z|)), \quad z \in R,
$$

and we may apply Lemma 5.2 to conclude that

$$
\|1-h\|_{\mathscr{D}}^{2} \leq c a^{2} \int_{R}\left|h^{\prime}\right|^{2} J(a(1-|z|)) d A .
$$

We can use the formula

$$
h(z)=\exp \left\{\int_{\partial D} \frac{e^{i \theta}+z}{e^{i \theta}-z} \log \left|h\left(e^{i \theta}\right)\right| \frac{d \theta}{2 \pi}\right\}
$$

and (7.5) (as well as the relations $\varepsilon^{s}<1$ and $|\Gamma|=s$ ) to check that

$$
\begin{aligned}
\left|h^{\prime}(z)\right|^{2} & <\frac{C}{(1-|z|)^{4}}\left(\log \left(\frac{1}{\varepsilon^{s}}\right)\right)^{2}|h(z)|^{2} \\
& <\frac{C}{(1-|z|)^{4}}\left(\log \left(\frac{1}{\varepsilon^{s}}\right)\right)^{2} \exp \left\{\frac{1}{\pi} \log \left(\frac{1}{\varepsilon^{s}}\right) \frac{1}{1-|z|}\right\}
\end{aligned}
$$

for some $C>0$ and all $z \in D$. The lemma follows on substituting this estimate into (7.6).

Proof of Theorem C. Let $N>0$. By hypothesis there exists $\varepsilon$ in $(0,1)$, an integer $n>1$, and $n$ equally spaced arcs $I_{1}, \ldots, I_{n}$ in $\partial D$, each of length $\frac{1}{n}$, such that both

$$
\frac{1}{\log n} \Theta\left(e^{-N}\right) \leq \frac{1}{N}
$$

and

$$
\Omega_{I_{k}}(\varepsilon) \log \frac{1}{\varepsilon} \geq N \text { for } k=1,2, \ldots, n .
$$

Clearly then, for each $k$ there exists a measurable set $\Delta_{k} \subset I_{k}$ with $w \leq \varepsilon$ on $\Delta_{k}$ and with $n\left|\Delta_{k}\right| \log \frac{1}{\varepsilon}=N$. Set $\Delta=\bigcup_{k=1}^{n} \Delta_{k}$ and associate the inner function $B$ to $\Delta$ as in $\S 2$. As usual $T$ will denote the composition operator $T: f \rightarrow f \circ B$, acting on our space $\mathscr{D}$. As in $\S 2$ we have $s=|\Delta|=|\Gamma|$. Since $s=|\Delta|=n\left|\Delta_{k}\right|$ (for each $k$ ) we have $\varepsilon^{s}=e^{-N}$. Now let $h$ be the outer function associated to $\varepsilon$ and $s$ as in (7.5). Lemmas 3.4 and 7.3 tell us that

$$
\inf _{p \in P^{2}(\mu)} \int\left|\chi_{D}-p\right|^{2} d \mu \leq a e^{-N / 2 \pi}+\kappa b\|T\|^{2} \Theta\left(e^{-N}\right) .
$$


We use Corollary 5.4 to estimate $\|T\|^{2}$. We may assume $n \geq \max \left(3, n_{0}\right)$ so that

$$
\begin{aligned}
\|T\|^{2} & \leq c \sup _{t>0} \frac{\log n F(t / \log n)}{F(t)} \\
& =c \sup _{t>0} \frac{\log n(t / \log n)^{2} J(t / \log n)}{t^{2} J(t)} \leq \frac{c}{\log n},
\end{aligned}
$$

the last inequality holding because $J$ is nondecreasing. On combining this with (7.7), we conclude that

$$
\inf _{p \in P^{2}(\mu)} \int\left|\chi_{D}-p\right|^{2} d \mu \leq a e^{-N / 2 \pi}+\kappa c b N^{-1} .
$$

Since $N$ is arbitrary, we have $\chi_{D} \in P^{2}(\mu)$.

\section{SharPNESS}

It is clear from Theorem D and Corollary 7.1 that Theorems $\mathrm{C}$ and $\mathrm{C}^{\prime}$ are quite sharp; in this section we consider Theorem 6.2 and Theorem $\mathrm{A}$. We inquire into the sharpness of our hypothesis

$$
\sup _{0<\varepsilon<t_{0}} \Omega(f(\varepsilon), \varepsilon) \log \frac{1}{\varepsilon}=+\infty,
$$

both with respect to the choice of $f$ (a function associated somewhat loosely to the weight $G$ ) and with respect to the factor $\log \frac{1}{\varepsilon}$. Let us begin with the latter.

Theorem 8.1. Suppose $\alpha>1,0<t_{0} \leq 1$ and let $f:\left(0, t_{0}\right) \rightarrow(0,2 \pi)$ be any continuous, strictly increasing function with $f(t) \rightarrow 0$ as $t \rightarrow 0$. Then there exists a positive $w \in L^{1}$ for which

$$
\sup _{0<\varepsilon<t_{0}} \Omega(f(\varepsilon), \varepsilon)\left(\log \frac{1}{\varepsilon}\right)^{\alpha}=+\infty,
$$

yet $\log w \in L^{1}$. Thus if $\nu$ is any measure carried by $D$ and $\mu=\nu+w d \theta$, then $P^{2}(\mu)$ does not split.

Proof. We use the proof of Proposition 6.3, with appropriate choices for the functions $p$ and $q$, to construct $w$. Begin by fixing $r<1$ and $\gamma>0$ so that $\alpha>\frac{1}{r}+\gamma$. Define $p$ and $q$ to be strictly increasing continuous functions mapping $[0,1]$ onto $[0,1]$ so that for $\varepsilon$ and $\delta$ sufficiently small $p(\varepsilon)=$ $(-\log \varepsilon)^{-1 / r}$ and $q(\delta)=\left[-\log f^{-1}(\delta)\right]^{-\gamma}$. Using these functions $p$ and $q$ and our chosen value of $r$, construct $g \in L^{1}$ as in the proof of Proposition 6.3 and set $w(x)=\exp \left(-\phi^{-1}(g(x))\right)$, where $p(\varepsilon)^{-r}=\phi\left(\log \frac{1}{\varepsilon}\right)$. Since $\log w(x)=$ $-g(x)$ when $w(x)$ is small, we have $\log w \in L^{1}$. Moreover, for $\varepsilon$ sufficiently close to 0 ,

$$
\Omega(f(\varepsilon), \varepsilon) \geq C p(\varepsilon) q(f(\varepsilon))=C\left(\log \frac{1}{\varepsilon}\right)^{-1 / r-\gamma} .
$$


Hence, by our choice of $r$ and $\gamma$ we have

$$
\sup _{0<\varepsilon<t_{0}} \Omega(f(\varepsilon), \varepsilon)\left(\log \frac{1}{\varepsilon}\right)^{\alpha}=+\infty .
$$

Next we consider sharpness with respect to $f$. Our original intuition was that the inner function $B$ defined in $\S 2$ should satisfy an inequality of the form $|B(z)| \leq|z|^{n^{b}}$ or even $|B(z)| \leq|z|^{b n}$ on some annulus (where $b$ is a possibly small positive number), thus improving the conclusion of Lemma 4.4. While we were unable to show this, we point out that if this were true, improved versions of our theorems would result. For example, in the case $\mu=(1-|z|)^{\alpha} d A+w d \theta$ either of the above estimates would allow one to choose $f(t)=t^{\gamma}$ (for any $\gamma>0)$ in Theorem A to conclude that splitting occurs if

$$
\sup _{0<\varepsilon<1} \Omega\left(\varepsilon^{\gamma}, \varepsilon\right) \log \frac{1}{\varepsilon}=+\infty \text {. }
$$

Whether or not Theorem A can be so improved, we will see that one cannot hope to do much better. Specifically, we have the following result.

Theorem 8.2. Let $0<\beta<1$ and suppose that $f(t)=\exp \left(-\left(\log \frac{1}{t}\right)^{\beta}\right)$. Then there exists a weight function $w$ (in fact $w=\chi_{E}$ where $E$ is a Cantor-like set) for which

$$
\sup _{0<\varepsilon<1} \Omega(f(\varepsilon), \varepsilon) \log \frac{1}{\varepsilon}=+\infty,
$$

yet $P^{2}\left((1-|z|)^{\alpha} d A+\chi_{E} d \theta\right)$ does not split, $\alpha>-1$.

The proof of Theorem 8.2 will use the necessary condition from Theorem 1.2 , with the choice $G(r)=(1-r)^{\alpha}$; without loss of generality we may assume that $\alpha \geq 0$. In particular, let $\mu=(1-|z|)^{\alpha} d A+\chi_{E} d \theta$ where $E$ is a proper closed subset of $\partial D$ (with $|E|>0$ ) having complementary arcs $\left\{I_{k}\right\}$. From Theorem 1.2 we see that if $E$ satisfies Carleson's condition, i.e. if

$$
\sum\left|I_{k}\right| \log \frac{1}{\left|I_{k}\right|}<\infty
$$

then $P^{2}(\mu)$ does not split.

Proof of Theorem 8.2. Let $\beta$ be fixed, $0<\beta<1$, and identify $\partial D$ with $(-\pi, \pi]$. We construct a Cantor-like set $E \subset[0,1] \subset(-\pi, \pi]$ by the usual procedure, as follows. Choose $\delta_{1}, 0<\delta_{1}<\frac{1}{2}$, and remove an open interval from the center of $[0,1]$ so as to leave two closed intervals $J_{1,1}$ and $J_{1,2}$, each of length $\delta_{1}$. From the center of each of $J_{1,1}$ and $J_{1,2}$, remove equallength open intervals, leaving four closed intervals $J_{2, k}, k=1,2,3,4$, each of length $\delta_{2}<\delta_{1} / 2$. Continuing in this way, we obtain at the $n$th stage $2^{n}$ closed intervals $J_{n, k}, k=1,2, \ldots, 2^{n}$, each of length $\delta_{n}<\delta_{n-1} / 2$. We put

$$
E_{n}=\bigcup_{k=1}^{2^{n}} J_{n, k} \text { and } E=\bigcap_{n=1}^{\infty} E_{n} \text {. }
$$


Clearly, $E_{n}$ has measure $a_{n}=2^{n} \delta_{n}$ and $1>a_{1}>a_{2}>\ldots$. Let us write $\tau=\lim _{n \rightarrow \infty} a_{n}$, so that $|E|=\tau$. We stipulate that $0<\tau<1$ and choose $a_{n}$ so that our conclusion holds.

First we claim that if $\Omega(\delta, \varepsilon)$ is associated to the weight $w=\chi_{E}$, then

$$
\Omega\left(\delta_{n}, \varepsilon\right)=1-\tau / a_{n}, \quad n=1,2, \ldots,
$$

provided $0<\varepsilon<1$. For this particular $w$ and any arc $I$ we have

$$
\Omega_{I}(\varepsilon)=1-\frac{|I \cap E|}{|I|}, \quad 0<\varepsilon<1 .
$$

We observe that the infimum $\Omega\left(\delta_{n}, \varepsilon\right)$ is attained by $\Omega_{I}(\varepsilon)$ when $I$ is any of the arcs $J_{n, k}, k=1,2, \ldots, 2^{n}$. Indeed, imagine $I$ to be any arc with $|I|=$ $\delta_{n}$. Then $I$ either intersects only one $J_{n, k}$ or else $I$ intersects two neighboring arcs $J_{n, k}$ and $J_{n, k+1}$ (but no others). Since $E \cap J_{n, k}$ and $E \cap J_{n, k+1}$ are exact replicas of each other under translation, and since $|I|=\delta_{n}$, it follows that $|I \cap E|$ is maximized by choosing $I=J_{n, k}$ (for any $k$ ) as desired. Now it is easy to see that

$$
\left|J_{n, k} \cap E_{n+i}\right|=2^{i} \delta_{n+i}=a_{n+i} / 2^{n}
$$

for $i=1,2, \ldots$, whence

$$
\Omega\left(\delta_{n}, \varepsilon\right)=\lim _{i \rightarrow \infty}\left(1-\frac{a_{n+i}}{2^{n} \delta_{n}}\right)=1-\frac{\tau}{a_{n}},
$$

and the claim is established.

Next consider the arcs complementary to $E$. There is one arc of length 1 $2 \delta_{1}$, two arcs of length $\delta_{1}-2 \delta_{2}$, and in general $2^{k-1}$ arcs of length $\delta_{k-1}-2 \delta_{k}$. Now $\delta_{k-1}-2 \delta_{k}=2^{-(k-1)}\left(a_{k-1}-a_{k}\right)$, and so the requirement that $E$ satisfy Carleson's condition is exactly equivalent to

$$
\begin{aligned}
& \sum_{k=2}^{\infty} k\left(a_{k-1}-a_{k}\right)<\infty, \\
& \sum_{k=2}^{\infty}\left(a_{k-1}-a_{k}\right) \log \frac{1}{\left(a_{k-1}-a_{k}\right)}<\infty .
\end{aligned}
$$

Now choose $p$ with $1<p-1<1 / \beta$ and set

$$
a_{n}=\tau+c \sum_{k=n+1}^{\infty} \frac{1}{k^{p}}, \quad n=1,2, \ldots,
$$

where $c>0$ is small enough that $a_{1}<1$. It follows from (8.1) that for some $d>0$,

$$
\Omega\left(\delta_{n}, \varepsilon\right)=\frac{c}{a_{n}} \sum_{k=n+1}^{\infty} \frac{1}{k^{p}} \geq d \frac{1}{n^{p-1}},
$$

$n=1,2, \ldots$. Moreover, $a_{k-1}-a_{k}=c k^{-p}$, and it follows (see (8.2)) that $E$ satisfies Carleson's condition. Therefore, $P^{2}\left((1-|z|)^{\alpha} d A+\chi_{E} d \theta\right)$ does not split. 
Next we choose $\gamma>0$ so that $\lambda \equiv p-1+\gamma<1 / \beta$ and consider the function $f(t)$ in the statement. Since $\lambda \beta<1$, we find that

$$
f\left(e^{-x^{\lambda}}\right)=e^{-x^{\lambda \beta}} \geq 2^{-x}
$$

for all $x$ exceeding some $N>0$. Let $\varepsilon_{n}=e^{-n^{\lambda}}, n=1,2, \ldots$, and note that since $a_{n}<1$,

$$
f\left(\varepsilon_{n}\right) \geq 1 / 2^{n}>a_{n} / 2^{n}=\delta_{n}, \quad n \geq N .
$$

We may apply Lemma 6.1 to find

$$
\Omega\left(f\left(\varepsilon_{n}\right), \varepsilon_{n}\right) \log \frac{1}{\varepsilon_{n}} \geq \frac{1}{2} \Omega\left(\delta_{n}, \varepsilon_{n}\right) \log \frac{1}{\varepsilon_{n}} \geq \frac{d}{2} \frac{1}{n^{p-1}} n^{\lambda}=\frac{d}{2} n^{\gamma}
$$

for $n \geq N$. It follows that

$$
\sup _{0<\varepsilon<1} \Omega(f(\varepsilon), \varepsilon) \log \frac{1}{\varepsilon}=+\infty,
$$

and the proof is complete.

If we limit our attention to weights $w=\chi_{E}$ where $E$ is a Cantor-like set, we even have a necessary condition for splitting.

Theorem 8.3. Let $E \subset \partial D$ be a Cantor-like set of positive measure and let $\mu=(1-|z|)^{\alpha} d A+\chi_{E} d \theta$ where $\alpha>-1$. If there exists $\beta, 0<\beta<\frac{1}{2}$, such that

$$
\sup _{0<\varepsilon<1} \Omega(f(\varepsilon), \varepsilon) \log \frac{1}{\varepsilon}<\infty,
$$

where $f(t)=\exp \left(-\left(\log \frac{1}{t}\right)^{\beta}\right)$, then $E$ satisfies Carleson's condition, hence $P^{2}(\mu)$ does not split.

Proof. The first three paragraphs of the proof of Theorem 8.2 apply perfectly well to our present Cantor-like set $E$ provided we allow $0<a_{1}<2 \pi$ and $0<\tau<2 \pi$. Again we have equation (8.1). Let $f(t)$ be as in the statement and define $\varepsilon_{n}$ by $f\left(\varepsilon_{n}\right)=\delta_{n}$ whenever $\delta_{n}<1$. Clearly

$$
\log \frac{1}{\varepsilon_{n}}=\log \frac{1}{f^{-1}\left(\delta_{n}\right)}=\left(\log \frac{1}{\delta_{n}}\right)^{1 / \beta} .
$$

Thus if $0<\varepsilon_{n}<1$, we see from (8.1) that

$$
\begin{gathered}
\left(a_{n}-\tau\right)\left(\log \frac{1}{\delta_{n}}\right)^{1 / \beta}<2 \pi \Omega\left(f\left(\varepsilon_{n}\right), \varepsilon_{n}\right) \log \frac{1}{\varepsilon_{n}} \\
\leq 2 \pi \sup _{0<\varepsilon<1} \Omega(f(\varepsilon), \varepsilon) \log \frac{1}{\varepsilon} \equiv M<\infty .
\end{gathered}
$$

We easily check that $\log \left(1 / \delta_{n}\right)>n / 2$ for $n>10$, and so $a_{n}-a_{n+1}<a_{n}-\tau<$ $M(2 / n)^{1 / \beta}, n>10$. Since $1 / \beta>2$, we see that the first sum in $(8.2)$ is finite. The second sum is finite as well because the function $t \log \frac{1}{t}$ increases with $t$ when $t$ is small. It follows that $E$ satisfies Carleson's condition. 


\section{CONCLUding REMARKS}

We mentioned earlier that Theorem $D$ improves a result of Trent [Tr1] by weakening the hypothesis on $G$. It should be noted that Trent obtains quite detailed information about the associated class of $P^{2}(\mu)$ spaces (where $\mu=$ $G d A+w d \theta$ ) with implications for the splitting problem arising as a by-product. Havin, Hruščëv and Nikol' skii [Kr2, p. 446] have observed that Trent's result can be strengthened in a different direction by weakening the hypothesis on $w$. The theorem so obtained extends the necessary condition of Theorem 1.2 to arbitrary $w$, with just a small modification of the proof (see the proof of Theorem 3.1 in $[\mathrm{Hr} 2]$ ):

Theorem. Suppose that

$$
\int_{1-\delta}^{1} \log \frac{1}{G(r)} d r<\infty \quad(\delta \text { small })
$$

(and that $G$ satisfies a mild regularity condition). If there exists a G-Carleson set $F$ of positive measure with $\log w \in L^{1}(F)$, then $P^{2}(\mu)$ does not split.

Let us hazard two conjectures, suggested by the above result, Theorems $\mathrm{C}$ and $\mathrm{D}$, and Theorem 1.2.

Conjecture 1. Suppose that

$$
\lim _{r \uparrow 1}(1-r) \log \frac{1}{G(r)}=0
$$

(and that $G$ perhaps satisfies some regularity conditions). If $\int_{F} \log w d \theta=-\infty$ for every $G$-Carleson set $F$ of positive measure, then $P^{2}(\mu)$ splits.

Conjecture 2. Suppose that $G$ satisfies

$$
\lim _{r \uparrow 1}(1-r) \log \frac{1}{G(r)}=+\infty
$$

(plus any necessary regularity conditions). If $\int_{I} \log w d \theta=-\infty$ for every arc $I$, then $P^{2}(\mu)$ splits.

We conclude with a remark on the role of symmetry in our methods. Let $\Delta$ be a measurable subset of $\partial D$ with $0<|\Delta|<2 \pi$. Define

$$
\sigma(\Delta)=\sup _{|z|=1 / 2} \frac{1}{2|\Delta|}\left|\int_{\Delta} \frac{1}{e^{i \theta}-z} d \theta\right|
$$

and note that $0<\sigma(\Delta)<1$. Alternatively, $|\Delta| \sigma(\Delta)$ is exactly the supremum of $|\log \Psi(z)-i| \Delta|/ 2|$ over the circle $|z|=\frac{1}{2}$, where $\Psi$ is the Cayley inner function belonging to $\Delta$. For the special $\Delta$ constructed in $\S 2$ we showed in the proof of Lemma 4.3 that $\sigma(\Delta) \leq 3 / n$. For general $\Delta$ as above one sees, as in Lemmas 4.3 and 4.4, that the associated inner function $B$ satisfies

$$
|B(z)| \leq|z|^{\log (1 / \sigma(\Delta))}, \quad \frac{1}{2}<|z|<1,
$$


provided $\sigma(\Delta)$ is small enough. The results of $\S \S 3$ and 5, with minor modification, easily yield the following:

General Distance Formula for $\mu=(1-|z|)^{\alpha} d A+w d \theta$. Suppose that $\Delta$ is a measurable subset of $\partial D$ with $0<|\Delta|<2 \pi$ and that $\sigma(\Delta)$ is defined by (9.1). Then

$$
\inf _{p \in P^{2}(\mu)} \int\left|\chi_{D}-p\right|^{2} d \mu \leq b\left(\int_{\Delta} w d \theta+\kappa\left(\log \frac{1}{\sigma(\Delta)}\right)^{-(1+\alpha)}\right)^{|\Delta| / 2 \pi},
$$

where $b$ and $\kappa$ are positive constants depending only on $\alpha$ and $w$.

As an immediate corollary we have

General Splitting Theorem for $\mu=(1-|z|)^{\alpha} d A+w d \theta$. Suppose there exists $a$ sequence $\left\{\Delta^{k}\right\}$ of measurable subsets of $\partial D$ such that

$$
\begin{aligned}
& \text { (i) }\left|\Delta^{k}\right| \log \int_{\Delta^{k}} w d \theta \rightarrow-\infty, \quad \text { and } \\
& \text { (ii) }\left|\Delta^{k}\right| \log \log \frac{1}{\sigma\left(\Delta^{k}\right)} \rightarrow+\infty
\end{aligned}
$$

as $k \rightarrow \infty$. Then $P^{2}(\mu)=P^{2}\left((1-|z|)^{\alpha} d A\right) \oplus L^{2}(w d \theta)$.

Condition (i) implies that as $k$ increases, $\Delta^{k}$ is increasingly concentrated where $w$ is small, whereas (ii) should tell us that $\Delta^{k}$ becomes progressively more symmetric in distribution. To understand this last point, recall that the estimate $\sigma(\Delta) \leq 3 / n$ in $\S 4$ depended on the rough symmetry of that special $\Delta$. For general $\Delta$, observe that a geometric series expansion of the Cauchy kernel gives

$$
|\Delta| \sigma(\Delta) \leq \sum_{k=1}^{\infty} \frac{1}{2^{k}}\left|\int_{\Delta} e^{i k \theta} d \theta\right|
$$

If, for example, $\Delta$ happens to be invariant under rotation by $2 \pi / n$, then one calculates that

$$
\int_{\Delta} e^{i k \theta} d \theta=0, \quad k=1,2, \ldots, n-1,
$$

and so $\sigma(\Delta) \leq 2^{-n+1}$, an even better estimate than $\sigma(\Delta) \leq 3 / n$. Conversely, if $\Delta$ lies in a single small arc, then $\sigma(\Delta)$ is close to 1 . Thus it may be worthwhile to study how the geometry of a general set $\Delta$ is reflected in the size of the Fourier coefficients of $\chi_{\Delta}$.

\section{REFERENCES}

[B1] J. Brennan, Point evaluations, invariant subspaces and approximation in the mean by polynomials, J. Funct. Anal. 34 (1979), 407-420.

[B2] _ Weighted polynomial approximation, quasianalyticity and analytic continuation, $\mathbf{J}$. Reine Angew. Math. 357 (1985), 23-50. 
[Ca] T. Carleman, Über die Approximation analytischer Funktionen durch lineare Aggregate von vorgegebenen Potenzen, Ark. Mat. Astronom. Fys. 17 (1923), 1-30.

[Cl] S. Clary, Quasi-similarity and subnormal operators, Doctoral Dissertation, Univ. of Michigan, 1973.

[Ha] W. Hastings, A construction of Hilbert spaces of analytic functions, Proc. Amer. Math. Soc. 74 (1979), 295-298.

[H] K. Hoffman, Banach spaces of analytic functions, Prentice-Hall, Englewood Cliffs, N. J., 1962.

[Hr1] S. Hruščèv, The removal of singularities for Cauchy integrals and an analogue of the HinčinOstrowski theorem for sequences of increasing functions, Dokl. Akad. Nauk SSSR 214 (1974), 524-527; English transl., Soviet Math. Dokl. 15 (1974), 198-202.

[Hr2] - The problem of simultaneous approximation and removal of singularities of Cauchytype integrals, Trudy Mat. Inst. Steklov 130 (1978), 124-195; English transl., Proc. Steklov Inst. Math. 130 (1979), no. 4, 133-203.

[Hr3] _ The Brennan alternative for measures with finite entropy, Izv. Akad. Nauk Armjan. SSR Ser. Math. 14 (1979), no. 3, 184-191, 234. (Russian, English summary)

[Ke] E. Kegegan, Simultaneous approximation in a disc, Izv. Akad. Nauk Armjan. SSR Ser. Math. 1 (1966), no. 5, 317-330. (Russian)

[Kd] M. Keldyš, Sur l'approximation en moyenne par polynomes des fonctions d'une variable complexe, Mat. Sb. 58 (1945), 1-20.

[Kr1] T. Kriete, On the structure of certain $H^{2}(\mu)$ spaces, Indiana Univ. Math. J. 28 (1979), 757-773.

[Kr2] _- Splitting and boundary behavior in certain $H^{2}(\mu)$ spaces, in Linear and Complex Analysis Problem Book (V. Havin et al., eds.), Lecture Notes in Math., vol. 1043, SpringerVerlag, New York, 1984.

[MS] B. MacCluer and J. Shapiro, Angular derivatives and compact composition operators on the Hardy and Bergman spaces, Canad. J. Math. 38 (1986), 878-906.

[Ne] R. Nevanlinna, Analytic functions, Grundlehren der Math. Wiss., Bd. 162, Springer-Verlag, Berlin, 1970.

[N] N. Nikol' skii, Selected problems of weighted approximation and spectral analysis, Trudy Mat. Inst. Steklov. 120 (1974), 271 pp.; English transl., Proc. Steklov Inst. Math. 120 (1974), $276 \mathrm{pp}$.

[RR] M. Rosenblum and J. Rovnyak, Change of variable formulas with Cayley inner functions, Topics in Functional Analysis, Adv. in Math. Suppl. Stud., vol. 3, Academic Press, New York, 1978, pp. 283-320.

[Ry] J. Ryff, Subordinate $H^{p}$ functions, Duke Math. J. 33 (1966), 347-354.

[Sc] H. Schwartz, Composition operators on $H^{p}$, Doctoral Dissertation, University of Toledo, Ohio, 1969.

[Sh] J. Shapiro, The essential norm of a composition operator, Ann. of Math. (2) 125 (1987), 375-404.

[Tr1] T. Trent, $H^{2}(\mu)$ spaces and bounded point evaluations, Pacific J. Math. 80 (1979), 279-292.

[Tr2] A characterization of $P^{2}(\mu) \neq L^{2}(\mu)$, J. Funct. Anal. 64 (1985), 163-177.

[V1] A. Vol' berg, Mean square completeness of polynomials beyond the scope of Szegö's theorem, Dokl. Akad. Nauk USSR 241 (1978), 521-527; English transl., Soviet Math. Dokl. 19 (1978), 877-881.

[V2] - Simultaneous approximation by polynomials on the circle and in the interior of the disc, Zap. Nauchn. Sem. Leningrad. Otdel. Mat. Inst. Steklov. (LOMI) 92 (1979), 60-84. (Russian)

[V3] - The logarithm of an almost analytic function is summable, Dokl. Akad. Nauk SSSR 265 (1982), 1297-1302; English transl., Soviet Math. Dokl. 26 (1982), 238-243. 
[VE] A. Vol' berg and B. Erikke, Summability of the logarithm of an almost analytic function and generalization of the Levinson-Cartwright theorem, Mat. Sb. (N.S.) 130(172) (1986), 335-348; English transl., Math. USSR-Sb. 58 (1987), 337-349.

[W] J. Wermer, On a class of normed rings, Ark. Mat. 2 (1953), 537-551.

Department of Mathematics, University of Virginia, Charlottesville, Virginia 22903 (Current address of T. L. Kriete III)

Department of Mathematics, University of South Carolina, Columbia, South CarOLINA 29208

Current address (B. D. MacCluer): Department of Mathematics, University of Richmond, Richmond, Virginia 23173 